\title{
Identification of a novel four-IncRNA signature as a prognostic indicator in cirrhotic hepatocellular carcinoma
}

\author{
Linkun Ma ${ }^{1}$, Cunliang Deng ${ }^{\text {Corresp. } 1}$ \\ ${ }^{1}$ Department of Infectious Diseases, The Affiliated Hospital of Southwestern Medical University, Luzhou, China \\ Corresponding Author: Cunliang Deng \\ Email address: dengcunl@swmu.edu.cn
}

Background. Many studies have shown that long noncoding RNAs ( IncRNA) are closely associated with the occurrence and development of various tumors and have the potential to be prognostic markers. Moreover, cirrhosis is an important prognostic risk factors in patients with liver cancer. Some studies have reported that IncRNA-related prognostic models have been used to predict overall survival (OS) and recurrence-free survival (RFS) in patients with hepatocellular carcinoma (HCC). However, no one has constructed a prognostic IncRNA model only in patients with cirrhotic HCC. Thus, it is necessary to screen novel potential IncRNA markers for improve the prognosis of cirrhotic HCC patients.

Methods. The probe expression profile dataset (GSE14520-GPL3921) from the Gene Expression Omnibus (GEO), which included 204 cirrhotic HCC samples, was reannotated and the IncRNA and mRNA expression dataset was obtained. The patients were randomly assigned to either the training set $(n=$ 103 ) and testing set $(n=100)$. Univariate cox regression and the least absolute shrinkage and selection operator (LASSO) model were applied to screen IncRNAs linked to the OS of cirrhotic HCC in the training set. The IncRNAs having significant correlation with OS were then selected and the multivariate Cox regression model was implemented to construct the prognostic score model. Whether or not this model was related to RFS in the training set was simultaneously determined. The testing set was used to validate the IncRNA risk score model. A risk score based on the IncRNA signature was used for stratified analysis of different clinical features to test their prognostic performance.The prognostic IncRNA-related protein genes were identified by the co-expression matrix of IncRNA-mRNA, and the function of these IncRNAs was predicted through the enrichment of these co-expression genes.

Results. The signature consisted of four IncRNAs:AC093797.1,POLR2J4,AL121748.1 and AL162231.4. The risk model was closely correlated with the OS of cirrhotic HCC in the training cohort, with a hazard ratio (HR) of 3.650 (95\% Cl: $1.761-7.566)$ and log-rank $P$ value of 0.0002 . Moreover, this model also showed favorable prognostic significance for RFS in the training set (HR: 2.392, 95\% Cl: 1.374-4.164, logrank $P=0.0015)$. The predictive performance of the four-IncRNA model for OS and RFS was verified in the testing set. Furthermore, the results of stratified analysis revealed that the four-IncRNA model was an independent factor in the prediction of OS and RFS of patients with clinical characteristics such as TNM (Tumor, Node, Metastasis system) stages I-II, Barcelona Clinic Liver Cancer (BCLC) stages 0-A, and solitary tumors in both the training set and testing set. The results of functional prediction showed that four IncRNAs may be potentially involve in multiple metabolic processes, such as amino acid, lipid, and glucose metabolism in cirrhotic HCC. 


\section{Identification of a novel four-IncRNA}

2 signature as a prognostic indicator in

3 cirrhotic hepatocellular carcinoma

4

5

6

7

8

9

10

11

12

13

14

15

16

17

18

19

20

21

22

23

24

25

26

27

28

29

30

31

32

33

34

35

36
Linkun $\mathrm{Ma}^{1}$, Cunliang Deng ${ }^{1}$

${ }^{1}$ Department of infectious disease, Affiliated Hospital of Southwestern Medical University, NO.25 Taiping street, Luzhou, 646000, China.

Corresponding Author:

Cunliang Deng ${ }^{1}$

Department of infectious disease, Affiliated Hospital of Southwestern Medical University, NO.25 Taiping street, Luzhou, 646000, China.

Email address: dengcun164@vip.sina.com 


\section{ABSTRACT}

38

39

40

41

42

43

44

45

46

47

48

49

50

51

52

53

54

55

56

57

58

59

60

61

62

63

64

65

66

67

68

69

70

71

Background. Many studies have shown that long noncoding RNAs (lncRNA) are closely associated with the occurrence and development of various tumors and have the potential to be prognostic markers. Moreover, cirrhosis is an important prognostic risk factors in patients with liver cancer. Some studies have reported that IncRNA-related prognostic models have been used to predict overall survival (OS) and recurrence-free survival (RFS) in patients with hepatocellular carcinoma (HCC). However, no one has constructed a prognostic lncRNA model only in patients with cirrhotic HCC. Thus, it is necessary to screen novel potential lncRNA markers for improve the prognosis of cirrhotic HCC patients.

Methods. The probe expression profile dataset (GSE14520-GPL3921) from the Gene Expression Omnibus (GEO), which included 204 cirrhotic HCC samples, was reannotated and the lncRNA and mRNA expression dataset was obtained. The patients were randomly assigned to either the training set $(n=103)$ and testing set $(n=100)$. Univariate cox regression and the least absolute shrinkage and selection operator (LASSO) model were applied to screen lncRNAs linked to the OS of cirrhotic HCC in the training set. The lncRNAs having significant correlation with OS were then selected and the multivariate Cox regression model was implemented to construct the prognostic score model. Whether or not this model was related to RFS in the training set was simultaneously determined. The testing set was used to validate the lncRNA risk score model. A risk score based on the lncRNA signature was used for stratified analysis of different clinical features to test their prognostic performance. The prognostic lncRNA-related protein genes were identified by the co-expression matrix of IncRNA -mRNA, and the function of these IncRNAs was predicted through the enrichment of these co-expression genes.

Results. The signature consisted of four lncRNAs: AC093797.1, POLR2J4, AL121748.1, and AL162231.4. The risk model was closely correlated with the OS of cirrhotic HCC in the training cohort, with a hazard ratio (HR) of 3.650 (95\% CI: 1.761-7.566) and log-rank P value of 0.0002 . Moreover, this model also showed favorable prognostic significance for RFS in the training set (HR: $2.392,95 \%$ CI: $1.374-4.164, \log$-rank $\mathrm{P}=0.0015$ ). The predictive performance of the fourIncRNA model for OS and RFS was verified in the testing set. Furthermore, the results of stratified analysis revealed that the four-lncRNA model was an independent factor in the prediction of OS and RFS of patients with clinical characteristics such as TNM (Tumor, Node, Metastasis system) stages I-II, Barcelona Clinic Liver Cancer (BCLC) stages 0-A, and solitary tumors in both the training set and testing set. The results of functional prediction showed that four lncRNAs may be potentially involve in multiple metabolic processes, such as amino acid, lipid, and glucose metabolism in cirrhotic HCC. 


\section{INTRODUCTION}

73 Worldwide, liver cancer remains among the top six prevalent carcinomas among males and

74 females from Global Cancer Statistics with an estimated 841,080 new cases and 781,631 deaths

75 occurring in 2018(International Agency for Research on Cancer, http://gco.iarc.fr/today/fact-

76 sheets-cancers).According to the US centers for disease control and prevention, the mortality of

77 liver cancer rose by 25 percent (from 5.3 per 100,000 to 6.6 per 100,000) between 2006 and

78 2015. (Centers for Disease Control and Prevention, 2018). Despite the existing treatment

79 methods, such as hepatectomy, liver transplantation, radiofrequency ablation, embolization

80 therapy, and molecule-targeted chemotherapy, high mortality and recurrence rates of liver

81 cancer, fundamentally, have not been changed, and new interventions to improve the poor

82 prognosis for patients with liver cancer is in continuing demand (Siegel, Miller \& Jemal, 2018).

83 The stratified management of patients with hepatocellular carcinoma (HCC) according to

84 specific clinical characteristics (e.g., using the Barcelona Clinic Liver Cancer staging system)

85 has improved the prognosis of patients (Villanueva, Hernandez-Gea \& Llovet, 2013).

86 Among the various pathological types of liver cancer, HCC is the most common, accounting for about $80 \%$ of all cases (DeSantis et al., 2014). The occurrence of HCC is closely related to liver fibrosis. Studies have reported that $80-90 \%$ of patients with HCC present liver fibrosis or cirrhosis (El-Serag, 2011). A recent meta-analysis of studies in cirrhotic patients with five liver diseases (hepatitis $\mathrm{B}$, hepatitis $\mathrm{C}$, primary biliary cholangitis, autoimmune hepatitis, and nonalcoholic steatohepatitis) showed that the incidence of liver cancer is significantly increased when liver disease develops into cirrhosis, indicating that cirrhosis is one of the crucial risk factors for HCC (Tarao et al., 2019). Patients with liver cancer, with or without cirrhosis, show different clinical characteristics, including differences in tumor size and prognostic factors (Techathuvanan et al., 2015). With the development of bioinformatics, many genomic biomarkers, including lncRNAs, have been explored. Three circulating lncRNAs, LINC00152, RP11-160H22.5 and XLOC014172, were found to could be candidate biomarkers towards diagnosis for hepatocellular carcinoma (Yuan et al., 2017). Hu et al 's meta-analysis showed that lncRNAs are correlated with the biological characteristics of gastric cancer, which may be a potential screening tool for the diagnosis of gastric cancer(Hu et al., 2017a). Hu et al confirmed that lncRNA-SVUGP2 expression in hepatocellular carcinoma tissues was associated with patient prognosis (Hu et al., 2017b). In addition, some lncRNAs have been found in serum to be biomarkers for tumor diagnosis. For instance, GIHCG has been found to be biomarkers for serum diagnosis of cervical cancer (Zhang et al., 2019). The lncRNAs are a type of noncoding RNAs longer than 200 nucleotides, which play various important roles in malignant tumors (Ma et al., 2017). The lncRNAs reportedly play a role in various biological processes, including cell proliferation, apoptosis, metastasis, and microvascular changes, and exhibit stem cell-like properties (Yuan et al., 2012; Wang et al., 2014; Cui et al., 2015; Ma et al., 2016). Several studies have demonstrated that lncRNAs contribute to the stratification of prognosis for patients with liver cancer (Liao et al., 2018; Zhao et al., 2018; Gu et al., 2018). For example, Yan et al 
112 the high-risk group was significantly lower than that of the low-risk group (Yan et al., 2019).

113 Currently, a lncRNAs signature is absent for stratification of prognosis in patients with cirrhotic

114 hepatocellular carcinoma. Some studies indicate that the knockdown of lncRNA SUMO1P3 can

115 inhibit the growth and invasion of hepatocellular carcinoma and enhance its radiotherapy

116 sensitivity (Zhou et al., 2019). This suggests that lncRNA can be a potential target for the

117 treatment of hepatocellular carcinoma. Therefore, this study aims to further explore the

118 prognostic biomarkers of the pathogenesis of cirrhotic HCC by analyzing microarray, and to

119 provide some potential targets for the biological treatment of HCC.

120

121

122

123

124

125

126

127

128

129

130

131

132

133

134

135

136

137

138

139

140

141

142

143

144

145

146

147

\section{MATERIALS \& METHODS}

\section{Data availability statement}

The following information was supplied regarding data availability:

Raw data were generated in the study. All original data in this study were downloaded from the public databases GEO (https://www.ncbi.nlm.nih.gov/geo/).

For the four-lncRNA signature identification, we used GSE14520 (https://www.ncbi.nlm.nih.gov /geo/query/acc.cgi?acc=GSE14520).

\section{Probe reannotation and preparation of gene expression profiles}

The GSE14520 expression profiles and clinical information analyzed in this study were derived from the GEO database (https://www.ncbi.nlm.nih.gov/geo/). To consider the expression and function of lncRNAs in the probes of HCC, the probes of the Affymetrix HT Human Genome U133A Array were reannotated to obtain the lncRNA and mRNA co-expression matrix using the following steps. Firstly, the Affymetrix HT Human Genome U133A Array probe was mapped to the gencode annotation file (Gencode.v29. transcripts, FASTA format,09/27/2018). Information about specific probe set annotation included the probe set ID, Ensemble ID, probe sequence, among other data. Secondly, the probe sets that were allocated to the Ensemble gene IDs in the gencode annotations were acquired. Human coding and noncoding gene annotation files (Homo_sapiens.GRCh38.95.chr.gtf.gz,GTF format,11/25/2018) from the Ensemble database were used to extract the matching information of gene ID and gene symbol. The Ensemble IDs of the probes were then assigned gene IDs, and only those annotated as "protein coding," "antisense," "sense intronic," "bidirectional promoter lncRNA," "lincRNA," "non-coding," "macro lncRNA," "processed transcript," "3' overlapping ncRNA," and "sense overlapping" were retained. In addition, probes that corresponded to multiple Ensemble IDs were removed. Finally, 12096 protein-coding transcripts and 610 lncRNA transcripts (different probe IDs may have corresponded to the same transcript) were reannotated.

The GSE14520 probe expression profile from the GEO database was annotated by the reannotated GPL3921 (Affymetrix HT Human Genome U133A Array), including 225 tumor samples and 220 paired non-tumor tissue samples. Among them, the survival information of 203 
148

149

150

151

152

153

154

155

156

157

158

159

160

161

162

163

164

165

166

167

168

169

170

171

172

173

174

175

176

177

178

179

180

181

182

183

184

185

patients was obtained from the dataset of GSE14520. If multiple probes corresponded to the same gene, the final expression of the gene was determined by the arithmetic mean of multiple probes. The gene expression profile of GSE14520 was normalized by the limma software package. Normalization was performed using the normalizeBetweenArrays function in the limma package (Bolstad et al., 2003). The inclusion criteria were as follows: pathologically confirmed HCC tissues, and patients with liver cirrhosis, and complete follow-up survival information, including overall survival (OS) and recurrence-free survival (RFS). Exclusion criteria included non-tumor tissues, absence of histological examination results, pathological results indicating cholangiocarcinoma, hepatocholangiocarcinoma, or secondary liver cancer, and missing OS or RFS information.

It contained $225 \mathrm{HCC}$ tissue samples and 220 adjacent non-tumor tissue samples. Out of 225 HCC samples, 221 with complete follow-up information (Survival status, Survival time, recurrence status and recurrence - free time) were included in the microarray. Of these 221 patients, 203 have cirrhosis. All 203 participants were patients who underwent radical resection of HCC at the Liver Cancer Institute and Zhongshan Hospital (Fudan University, Shanghai, China) between 2002 and 2003. Of the 203 participants, 177 were male and 26 were female .The mean age of all patients in the dataset was 51.3 years . Regarding the TNM stage of HCC, 153 of the 203 patients were in stage I or II, and 48 were in stage III. Only 2 patients had no documented cancer stage.

\section{Data and clinical characteristics}

All 204 patients with liver cancer from the GSE14520 of the GEO database had complete survival information and were included in the study. These patients were randomly divided into the training set $(n=103)$ and testing set $(n=100)$ using the $R$ caret software package. The clinical parameters of the two groups of patients are presented in Table 1.

\section{Construction of IncRNA expression-based prognostic signature}

Univariate Cox regression analysis was used to screen for significant prognostic lncRNAs in the training set. A $P$ value $<0.05$ was selected as the threshold for candidate lncRNAs. Candidate prognostic lncRNAs were further reduced by least absolute shrinkage and selection operator (LASSO) regression. The principle of LASSO regression is to eliminate some variables through penalty rules, and ultimately leave all potential predictors with a non-zero coefficient (Gao, Kwan \& Shi, 2010). The penalty argument lambda was determined by the cross-validation method using the R glmnet software package. Lambda.min, the lambda value corresponding to the minimum value of the cross-validation error mean, was identified to determine the potential prognostic lncRNAs (Tibshirani, 1997). Each "significant" lncRNA obtained in the above steps were then fitted into a multivariate Cox regression model, and lncRNAs associated with independent prognostic criteria were selected. The prognostic risk formulas were formed, based on the expression level of those remaining eligible lncRNAs multiplied by the multivariate Cox 
186 regression coefficient. Risk scores for each sample were calculated using the risk score formula, 187 and patients were divided into high-risk and low-risk groups based on the median risk score of 188 the training cohort as the cutoff value. The same formulas and cutoff values were used to 189 calculate the risk score and risk grouping for patients in the testing set (Zhao et al., 2018). A 190 Kaplan-Meier survival curve and log-rank test were used to analyze the overall survival (OS) 191 and recurrence-free survival (RFS) of patients in the high-risk and low-risk groups in both the 192 training and testing set (Li et al., 2018). A receiver operating characteristic (ROC) curve plotted 193 with the survivalROC software package (version 1.0.3) was adopted to evaluate the specificity 194 and sensitivity of the survival prediction (Heagerty, Lumley \& Pepe, 2000). Both ROC and 195 Kaplan-Meier survival curves were constructed with the R studio software (version 3.5.1). A $P$ 196 value $<0.05$ was deemed statistically significant.

197

198

199

In order to obtain the IncRNA-mRNA co-expression pair, we conducted Pearson correlation

200

201

202

203

204

205

206

207

208

209

210

211

212

213

214

215

216

218

219

220 analyses between the four lncRNAs identified and the expression profile of the protein-coding genes of the discovery set. The protein-coding genes with a correlation coefficient $>0.4$ and $P<$ 0.001 were recognized as the four lncRNA-associated genes. The correlated mRNAs were evaluated by functional enrichment analysis to explore the functions of the four prognostic lncRNAs using the clusterProfiler (version 3.10.1) and org.Hs.eg.db (version 3.7.0) package (Yu et al., 2012). Significantly enriched Gene Ontology (GO) terms and Kyoko Encyclopedia of Genes and Genomes (KEGG) pathways with a $P$ value $<0.05$ and $q$ value $<0.05$ were visualized using the R studio (version 3.5.1) software.

\section{Statistical analysis}

The prognostic ability of the four-lncRNA signature under different clinical parameters was analyzed by Kaplan-Meier survival analysis, to determine which population is most suited for this prognostic model. The survival curve was drawn using the $\mathrm{R}$ survminer software package. Statistical analyses were conducted using the SPSS software (version 24.0) and R studio (version 3.5.1).

\section{RESULTS}

\section{Construction of risk prognostic scoring system in the training set}

The exploration process of this study is shown in figure 1. Firstly, 610 lncRNAs were initially screened in the training set (Table S1), and 35 potential OS-related lncRNAs were obtained by a univariate Cox risk regression model (Table S2) $(P<0.05)$. The remaining lncRNAs were 
221 further selected using LASSO regression analysis, and cross-validation was used to select the

222

223

224

225

226

227

228

229

230

231

232

233

234

235

236

237

238

239

240

241

242

243

244

245

246

247

248

249

250

251

252

253

254

255

256

257

258

259

penalty parameters (Fig. 2A-B). Five lncRNAs were identified by lambda.min value (Table S3). The IncRNAs obtained in the above steps were inserted into the multivariate Cox regression model. The expression values of four independent prognostic lncRNAs $(P$ value $<0.05)$ and their correlation coefficients in a multivariate regression model were then used to construct prognostic signatures. Detailed information and the significance of survival prediction by the four lncRNAs are presented in Table 2.

Risk score $=($ expression quantity of AC093797.1 $\times-0.4818)+($ expression quantity of AL121748.1 $\times 0.4404)+($ expression quantity of AL162231.4 $\times 1.2845)+($ expression quantity of POLR2J4 $\times-1.5170)$.

The risk score formula in the training set was used to calculate the risk score of each patient. The median score for all patients in the training set was deemed the cutoff value, and facilitated the division of patients into a high-risk group $(\mathrm{n}=51)$ and low-risk group $(\mathrm{n}=52)$ (Table S4). Patients of the training group were ranked in ascending order of risk score (Fig.3A). Moreover, the expression profiles of the four lncRNAs were plotted on a heatmap (Fig. 3B). In addition, a scatter plot was constructed to show the OS status and recurrence status of patients (Fig. 3C-D). The high-risk group had a worse prognosis than the low-risk group in terms of OS and RFS. The prognosis of the high-risk group was compared with that of the low-risk group using KaplanMeier curve analysis. The results showed that the OS (HR: 3.650, 95\% CI: 1.761-7.566, logrank $P=0.0002)$ and RFS (HR: $2.392,95 \%$ CI: $1.374-4.164$, log-rank $P=0.0015)$ of high-risk patients were significantly lower than those of low-risk patients (Fig. 4A-B). Furthermore, according to the four-lncRNA signature, the area under the curve of time-dependent ROC analysis of the 5-year OS and RFS predicted in the training set reached 0.839 and 0.715 , respectively (Fig. 4C-D).

\section{Prognostic performance of the four-IncRNA signature in the testing set}

To further assess the prognostic significance of the four-lncRNA signature, internal validation was performed in a group of 100 patients from the testing set. The established formula for the training set was used to calculate the patient risk score for the testing set. The same cutoff value used for the training set was also used to divide the testing set into high-risk $(n=44)$ and lowrisk cohorts $(\mathrm{n}=56)$ (Table S5). Kaplan-Meier survival analysis was also performed in the testing set. As shown in Fig. 5A-B, the four-lncRNA signature showed favorable prognostic value in differentiating the risk stratification of death (HR: $2.475,95 \%$ CI: $1.347-4.547$, log-rank $P=0.0015)$ and recurrence (HR: $2.245,95 \%$ CI: $1.349-3.736, \log$-rank $P=0.0014)$ in patients. The ROC curve was used to analyze the predictive power of risk models in the validation group for 5-year OS (Fig. 5C) and RFS (Fig. 5D). The OS and RFS of patients in the testing group were plotted (Fig. 5E-F). These results suggested that the four-lncRNA signature in patients with cirrhotic HCC has considerable potential in predicting OS and RFS.

Peer) reviewing PDF | (2019:04:36386:1:1:REVIEW 21 Jun 2019) 
260

261

262

263

264

265

266

267

268

269

270

271

272

273

274

275

276

277

278

279

280

281

282

283

284

285

286

287

288

289

290

291

292

293

294

295

296

297

\section{Stratification analysis}

To explore the different clinical characteristics for which the four-lncRNA model is applicable, we conducted subgroup analyses of OS and RFS on patients of both the training set and testing set. The risk grouping principle was based on the median four-lncRNA risk score of the training set. Subgroup analyses combining the training and validation sets showed that the risk score model based on the four-lncRNA signature was more appropriate for patients with the following characteristics: BCLC stages 0-A, solitary tumors, and Tumor, Node, Metastasis system (TNM) stages I-II. The results of subgroup analyses of the OS and RFS are shown in Tables 3 and 4, respectively. Kaplan-Meier curves were constructed for both high-risk and low-risk patients with TNM stages I-II, solitary tumors, and BCLC stages 0-A, to analyze the prognostic value of risk models in OS (Fig. 6A-F) and RFS of cirrhotic HCC (Fig. 7A-F). Among the patients with the three aforementioned clinical characteristics, the OS (Fig. 6A $(P=0.00021)$, Fig. $6 \mathrm{~B}(P=$ $0.0015)$, Fig. 6C $(P=0.0012)$, Fig. 6D $(P=0.0043)$, Fig. 6E $(P=0.00033)$, and Fig. 6F $(P=$ $0.0019))$ and RFS (Fig. 7A $(P=0.0018)$, Fig. 7B $(P=0.0019)$, Fig. 7C $(P=0.0028)$, Fig. 7D $(P$ $=0.00061)$, Fig. 7E $(P=0.0023)$, and Fig. 7F $(P=0.0018))$ of high-risk patients were worse than those of low-risk patients.

\section{Functional enrichment analyses}

Firstly, the co-expression matrix of the four lncRNAs and 12,100 protein-coding genes were extracted from 204 patients with tumor tissue gene expression profile data from GSE14520. The correlation between lncRNAs and protein-coding genes was analyzed. The significantly correlated protein-coding genes (Pearson coefficient $>0.4, P<0.001$ ) were used for $\mathrm{GO}$ analysis (Table S6) and KEGG enrichment analysis (Table S7) to determine the potential mechanism of the four lncRNAs in regulating HCC. Functional enrichment analysis was conducted with the R org.Hs.eg.db (version 3.7.0) and clusterProfiler (version 3.10.1) package (Yu et al., 2012). The first 25 significant GO terms $(P<0.05, q<0.05)$ (Fig. 8A) and first 25 significant KEGG pathways $(P<0.05, q<0.05)$ (Fig. 8B) were charted using the $\mathrm{R}$ software (version 3.5.1). The results of GO analysis revealed that four-lncRNA-related functions showed enrichment in protein-coding genes involved in a large group of GO terms, including those associated with transmembrane transport, redox reactions, and fatty acid metabolism. Furthermore, KEGG analysis showed significant associations with a series of enriched pathways, including the peroxisome proliferator-activated receptor (PPAR) signaling pathway, amino acid metabolism, fatty acid metabolism, and chemical carcinogenesis.

\section{DISCUSSION}

Currently, the popularity and wide application of gene microarray technology has presented considerable convenience to researchers who attempt to develop prognostic biomarkers of tumors. A large number of studies have confirmed that lncRNAs play an indispensable role in 
298 the proliferation, metastasis, metabolic regulation, and drug resistance of various tumors (Pan et 299 al., 2016; Kia et al., 2019; Liu et al., 2019; Schwarzenbacher et al., 2019; Zheng et al., 2019). 300 Therefore, their performance in determining the prognosis of various tumors has drawn 301 widespread interest. The significance of prognostic signatures based on lncRNA expression has 302 been demonstrated in various tumors, including those of head and neck squamous cell carcinoma 303 (Cao et al., 2017), gastric cancer (Song et al., 2017), breast cancer (Li et al., 2018), cervical 304 squamous cell carcinoma (Mao et al., 2018), and HCC (Song et al., 2017). For example, RGMB305 AS1 plays an anti-tumor role by regulating a variety of biological processes in HCC cells (Sheng 306 et al., 2018). Many unknown functional lncRNAs are yet to be explored. Moreover, cirrhosis of 307 the liver is often associated with liver cancer, and more than $80 \%$ of patients with liver cancer 308 have cirrhosis ( Affo, Yu \& Schwabe, 2017). To explore new prognostic lncRNAs in patients 309 with cirrhotic HCC, patients with HCC and liver cirrhosis were included as subjects of the 310 present study, and the lncRNA expression profile and clinical information were extracted from 311 the GEO database for a comprehensive analysis.

312 We developed a prognosis formula for cirrhotic HCC based on the four lncRNAs, including 313 AC093797.1, AL121748.1, AL162231.4, and POLR2J4, and verified it in the testing set. Patients 314 were divided into high-risk and low-risk groups according to the prognostic signature score. 315 Kaplan-Meier analysis confirmed that the four-lncRNA signature has favorable OS and RFS 316 prediction ability and could be considered a new prognostic biomarker. Stratified analysis was 317 used to assess the prognostic performance of this signature in patients with different clinical 318 characteristics. We found that the four-lncRNA signature was significantly correlated with OS 319 and RFS in patients with specific characteristics, including TNM stages I-II, solitary tumors, and 320 321 BCLC stages 0-A. The selection of appropriate treatment methods is of prime importance for improving the prognosis of patients with early liver cancer (Vitale et al., 2017). In addition, the results of a relevant meta-analysis showed that liver transplantation had a higher OS rate and lower recurrence rate than hepatectomy among patients with liver cancer and Child-TurcottePugh class A cirrhosis (Zheng et al., 2014). Although liver transplantation can achieve a better prognosis, it is especially important to be selective in determining the candidates for this procedure, owing to a shortage of donor livers. Our four-lncRNA label can help clinicians to predict and stratify the prognosis of patients after surgery, and implement reasonable treatment programs.

330 Among the four lncRNAs, AC093797.1 and POLR2J4 were risk factors for liver cancer, whereas the other two were protective factors (AL121748.1 and AL162231.4). With the exception of POLR2J4, the lncRNAs were deemed prognostic markers of liver cancer for the first time, to our knowledge. Their function in HCC is unclear, and we determined their potential biological function through function enrichment analysis of related proteins. The GO enrichment analysis showed that the four lncRNA-related protein-coding genes were mainly involved in lipid metabolism and glycolysis, including fatty acid ligase activity, long-chain fatty acid-CoA ligase 337 activity, oxidoreductase activity, and CoA ligase activity, among other processes. The KEGG pathway analysis showed that most genes were enriched in fatty acid and amino acid 
338

339

340

341

342

343

344

345

346

347

348

349

350

351

352

353

354

355

356

357

358

359

360

361

362

363

364

365

366

367

368

369

370

371

372

373

374

375

metabolism. This indicates that the four lncRNAs may be potentially involved in tumorigenesis and the development of tumors through the regulation of metabolism. At present, tumor metabolism is one of the more popular topics in tumor research. Some studies have reported that lncRNAs can affect tumor metabolism through a variety of methods, such as by regulating oncogenes, tumor suppressor factors, and crucial transcription factors to affect tumor growth (Liu et al., 2019). Thus, IncRNAs are expected to be targets for future tumor treatment. The specific mechanism of the four IncRNAs in the present study in the regulation of tumor metabolism still needs to be further clarified in cell and animal experiments.

This study is a preliminary study that explored the prognosis of cirrhotic HCC. The data of only a single liver cancer cohort were included in the GEO database that was used to develop and verify the lncRNA signature. In addition, an independent external validation set of larger samples to verify the reliability of the model is lacking. Moreover, further experimental studies to verify the potential functions of the four IncRNAs are also lacking. Nevertheless, the fourlncRNA signature developed, based on the expression of lncRNA from the GEO database, has the potential to become a new and highly effective biomarker for patients with cirrhotic HCC. The present study lays the foundation for further exploration of prognostic markers in patients with early HCC.

\section{CONCLUSIONS}

In summary, we identified four potential lncRNAs biomarkers associated with prognosis in cirrhotic HCC, and constructed a risk score model. Patients in the high-risk group had lower overall survival and relapse-free survival than those in the low-risk group. Based on stratification analysis, the prognostic risk model might have ability to predict OS and RFS in cirrhotic HCC patients with BCLC stages 0-A, solitary tumors, and Tumor, Node, Metastasis system (TNM) stages I-II. Moreover, the four new lncRNAs identified could be used as potential therapeutic targets.

\section{REFERENCES}

Affo, S., Yu, L.X., and Schwabe, R.F. 2017. The Role of Cancer-Associated Fibroblasts and Fibrosis in Liver Cancer. ANNUAL REVIEW OF PRATHOLOGY 12:153-186. doi:10.1146/annurev-pathol-052016-100322

Bolstad, B.M., Irizarry, R.A., Astrand, M., and Speed, T.P. 2003. A comparison of normalization methods for high density oligonucleotide array data based on variance and bias. BIOINFORMATICS 19:185-193.

Cao, W., Liu, J.N., Liu, Z., Wang, X., Han, Z.G., Ji, T., Chen, W.T., and Zou, X. 2017. A threelncRNA signature derived from the Atlas of ncRNA in cancer (TANRIC) database predicts the survival of patients with head and neck squamous cell carcinoma. ORAL ONCOLOGY 65:94101. doi:10.1016/j.oraloncology.2016.12.017 
376 Cui, M., Xiao, Z., Wang, Y., Zheng, M., Song, T., Cai, X., Sun, B., Ye, L., and Zhang, X. 2015. 377 Long noncoding RNA HULC modulates abnormal lipid metabolism in hepatoma cells through 378 an miR-9-mediated RXRA signaling pathway. CANCER RESEARCH 75:846-857. 379 doi:10.1158/0008-5472.CAN-14-1192

380 DeSantis, C.E., Lin, C.C., Mariotto, A.B., Siegel, R.L., Stein, K.D., Kramer, J.L., Alteri, R., 381 Robbins, A.S., and Jemal, A. 2014. Cancer treatment and survivorship statistics, 2014. $C A$ : $A$ 382 CANCER JOURNAL FOR CLINICIANS 64:252-271. doi:10.3322/caac.21235

383 El-Serag, H.B. 2011. Hepatocellular carcinoma. NEW ENGLAND JOURNAL OF MEDICINE 384 365:1118-1127. doi:10.1056/NEJMra1001683

Gao, J., Kwan, P.W., and Shi, D. 2010. Sparse kernel learning with LASSO and Bayesian inference algorithm. NEURAL NETWORKS 23:257-264. doi:10.1016/j.neunet.2009.07.001

387

388

389

390

391

392

393

394

395

396

397

398

399

400

401

402

403

404

405

406

407

408

409

410

411

412

413

414

415

416

417

418

419

420

421

Heagerty, P.J., Lumley, T., and Pepe, M.S. 2000. Time-dependent ROC curves for censored survival data and a diagnostic marker. BIOMETRICS 56:337-344.

Hu, Q.Y., Zhao, Z.Y., Li, S.Q., Li, L., and Li, G.K. 2017a. A meta-analysis: The diagnostic values of long non-coding RNA as a biomarker for gastric cancer. MOLECULAR AND CLINICAL ONCOLOGY 6:846-852. 10.3892/mco.2017.1227

Hu, J., Song, C., Duan, B., Zhang, X., Li, D., Zhu, L., and Gao, H. 2017b. LncRNA-SVUGP2 suppresses progression of hepatocellular carcinoma. ONCOTARGET 8:97835-97850. 10.18632/oncotarget.18279

Gu, J., Zhang, X., Miao, R., Ma, X., Xiang, X., Fu, Y., Liu, C., Niu, W., and Qu, K. 2018. A three-long non-coding RNA-expression-based risk score system can better predict both overall and recurrence-free survival in patients with small hepatocellular carcinoma. AGING (ALBANY NY) 10:1627-1639. 10.18632/aging.101497

Kia, V., Mortazavi, Y., Paryan, M., Biglari, A., and Mohammadi-Yeganeh, S. 2019. Exosomal miRNAs from highly metastatic cells can induce metastasis in non-metastatic cells. LIFE SCIENCES 220:162-168. doi:10.1016/j.lfs.2019.01.057

Li, J., Wang, W., Xia, P., Wan, L., Zhang, L., Yu, L., Wang, L., Chen, X., Xiao, Y., and Xu, C. 2018. Identification of a five-lncRNA signature for predicting the risk of tumor recurrence in patients with breast cancer. INTERNATIONAL JOURNAL OF CANCER 143:2150-2160. doi:10.1002/ijc.31573

Liao, X., Yang, C., Huang, R., Han, C., Yu, T., Huang, K., Liu, X., Yu, L., Zhu, G., Su, H., Wang, X., Qin, W., Deng, J., Zeng, X., Ye, X., and Peng, T. 2018. Identification of Potential Prognostic Long Non-Coding RNA Biomarkers for Predicting Survival in Patients with Hepatocellular Carcinoma. CELLULAR PHYSIOLOGY AND BIOCHEMISTRY 48:1854-1869. doi:10.1159/000492507

Liu, H., Luo, J., Luan, S., He, C., and Li, Z. 2019. Long non-coding RNAs involved in cancer metabolic reprogramming. CELLULAR AND MOLECULAR LIFE SCIENCES 76:495-504. doi:10.1007/s00018-018-2946-1

Ma, Y., Huang, D., Yang, F., Tian, M., Wang, Y., Shen, D., Wang, Q., Chen, Q., and Zhang, L. 2016. Long Noncoding RNA Highly Upregulated in Liver Cancer Regulates the Tumor Necrosis Factor-alpha-Induced Apoptosis in Human Vascular Endothelial Cells. DNA AND CELL BIOLOGY 35:296-300. doi:10.1089/dna.2015.3203

Ma, Z., Huang, H., Xu, Y., He, X., Wang, J., Hui, B., Ji, H., Zhou, J., and Wang, K. 2017. Current advances of long non-coding RNA highly upregulated in liver cancer in human tumors. ONCOTARGETS AND THERAPY 10:4711-4717. doi:10.2147/OTT.S136915

Mao, X., Qin, X., Li, L., Zhou, J., Zhou, M., Li, X., Xu, Y., Yuan, L., Liu, Q.N., and Xing, H.

Peer] reviewing PDF | (2019:04:36386:1:1:REVIEW 21 Jun 2019) 
422 2018. A 15-long non-coding RNA signature to improve prognosis prediction of cervical

423

424

425

426

427

428

429

430

431

432

433

434

435

436

437

438

439

440

441

442

443

444

445

446

447

448

449

450

451

452

453

454

455

456

457

458

459

460

461

462

463

464

465

466

467 squamous cell carcinoma. GYNECOLOGIC ONCOLOGY 149:181-187.

doi:10.1016/j.ygyno.2017.12.011

Pan, J., Li, X., Wu, W., Xue, M., Hou, H., Zhai, W., and Chen, W. 2016. Long non-coding RNA UCA1 promotes cisplatin/gemcitabine resistance through CREB modulating miR-196a-5p in bladder cancer cells. CANCER LETTERS 382:64-76. doi:10.1016/j.canlet.2016.08.015

Schwarzenbacher, D., Klec, C., Pasculli, B., Cerk, S., Rinner, B., Karbiener, M., Ivan, C., Barbano, R., Ling, H., Wulf-Goldenberg, A., Stanzer, S., Rinnerthaler, G., Stoeger, H., Bauernhofer, T., Haybaeck, J., Hoefler, G., Jahn, S.W., Parrella, P., Calin, G.A., and Pichler, M. 2019. MiR-1287-5p inhibits triple negative breast cancer growth by interaction with phosphoinositide 3-kinase $\mathrm{CB}$, thereby sensitizing cells for PI3Kinase inhibitors. BREAST CANCER RESEARCH 21:20. doi:10.1186/s13058-019-1104-5

Sheng, N., Li, Y., Qian, R., and Li, Y. 2018. The clinical significance and biological function of lncRNA RGMB-AS1 in hepatocellular carcinoma. BIOMEDICINE \& PHARMACOTHERAPY 98:577-584. doi:10.1016/j.biopha.2017.12.067

Siegel, R.L., Miller, K.D., and Jemal, A. 2018. Cancer statistics, 2018. CA: A CANCER JOURNAL FOR CLINICIANS 68:7-30. doi:10.3322/caac.21442

Song, P., Jiang, B., Liu, Z., Ding, J., Liu, S., and Guan, W. 2017. A three-lncRNA expression signature associated with the prognosis of gastric cancer patients. CANCER MEDICINE 6:11541164. doi:10.1002/cam4.1047

Tarao, K., Nozaki, A., Ikeda, T., Sato, A., Komatsu, H., Komatsu, T., Taguri, M., and Tanaka, K. 2019. Real impact of liver cirrhosis on the development of hepatocellular carcinoma in various liver diseases-meta-analytic assessment. CANCER MEDICINE 8:1054-1065. doi:10.1002/cam4.1998

Techathuvanan, K., Srisajjakul, S., Pongpaibul, A., Limsrichamrern, S., Charatcharoenwitthaya, P., Chainuvati, S., Tanwandee, T., and Chotiyaputta, W. 2015. Comparison between disease free survival of hepatocellular carcinoma after hepatic resection in chronic hepatitis B patients with or without cirrhosis. JOURNAL OF THE MEDICAL ASSOCIATION OF THAILAND 98:334342 .

Tibshirani, R. 1997. The lasso method for variable selection in the Cox model. STATISTICS IN MEDICINE 16:385-395.

Villanueva, A., Hernandez-Gea, V., and Llovet, J.M. 2013. Medical therapies for hepatocellular carcinoma: a critical view of the evidence. NATURE REVIEWS GASTROENTEROLOGY \& HEPATOLOGY 10:34-42. doi:10.1038/nrgastro.2012.199

Vitale, A., Peck-Radosavljevic, M., Giannini, E.G., Vibert, E., Sieghart, W., Van Poucke, S., and Pawlik, T.M. 2017. Personalized treatment of patients with very early hepatocellular carcinoma. JOURNAL OF HEPATOLOGY 66:412-423. doi:10.1016/j.jhep.2016.09.012

Wang, F., Yuan, J.H., Wang, S.B., Yang, F., Yuan, S.X., Ye, C., Yang, N., Zhou, W.P., Li, W.L., Li, W., and Sun, S.H. 2014. Oncofetal long noncoding RNA PVT1 promotes proliferation and stem cell-like property of hepatocellular carcinoma cells by stabilizing NOP2. HEPATOLOGY 60:1278-1290. doi:10.1002/hep.27239

Yan, J., Zhou, C., Guo, K., Li, Q., and Wang, Z. 2019. A novel seven-lncRNA signature for prognosis prediction in hepatocellular carcinoma. JOURNAL OF CELLULAR BIOCHEMISTRY 120:213-223. doi:10.1002/jcb.27321

Yu, G., Wang, L.G., Han, Y., and He, Q.Y. 2012. clusterProfiler: an R package for comparing biological themes among gene clusters. OMICS-A JOURNAL OF INTEGRATIVE BIOLOGY 
468 16:284-287. doi:10.1089/omi.2011.0118

469 Yuan, S.X., Yang, F., Yang, Y., Tao, Q.F., Zhang, J., Huang, G., Yang, Y., Wang, R.Y., Yang, 470 S., Huo, X.S., Zhang, L., Wang, F., Sun, S.H., and Zhou, W.P. 2012. Long noncoding RNA 471 associated with microvascular invasion in hepatocellular carcinoma promotes angiogenesis and 472 serves as a predictor for hepatocellular carcinoma patients' poor recurrence-free survival after 473 hepatectomy. HEPATOLOGY 56:2231-2241. doi:10.1002/hep.25895

474 Yuan, W., Sun, Y., Liu, L., Zhou, B., Wang, S., and Gu, D. 2017. Circulating LncRNAs Serve as 475 Diagnostic Markers for Hepatocellular Carcinoma. CELLULAR PHYSIOLOGY AND 476 BIOCHEMISTRY 44:125-132. 10.1159/000484589

477 Zhang, X., Mao, L., Li, L., He, Z., Wang, N., and Song, Y. 2019. Long noncoding RNA GIHCG 478 functions as an oncogene and serves as a serum diagnostic biomarker for cervical cancer. 479 JOURNAL OF CANCER 10:672-681. 10.7150/jca.28525

480 Zhao, Q.J., Zhang, J., Xu, L., and Liu, F.F. 2018. Identification of a five-long non-coding RNA 481 signature to improve the prognosis prediction for patients with hepatocellular carcinoma. 482 WORLD JOURNAL OF GASTROENTEROLOGY 24:3426-3439. doi:10.3748/wjg.v24.i30.3426 Zheng, Y., Lu, S., Xu, Y., and Zheng, J. 2019. Long non-coding RNA AGAP2-AS1 promotes the proliferation of glioma cells by sponging miR-15a/b-5p to upregulate the expression of HDGF and activating Wnt/beta-catenin signaling pathway. INTERNATIONAL JOURNAL OF BIOLOGICAL MACROMOLECULES 128:521-530. doi:10.1016/j.ijbiomac.2019.01.121

Zheng, Z., Liang, W., Milgrom, D.P., Zheng, Z., Schroder, P.M., Kong, N.S., Yang, C., Guo, Z., and He, X. 2014. Liver transplantation versus liver resection in the treatment of hepatocellular carcinoma: a meta-analysis of observational studies. TRANSPLANTATION 97:227-234. doi:10.1097/TP.0b013e3182a89383

Centers for Disease Control and Prevention. Liver and Intrahepatic Bile Ducts Cancers, United States-2006-2015. USCS data brief, no 5. Atlanta, GA: Centers for Disease Control and Prevention; 2018.

International Agency for Research on Cancer, World Health Organization. Cancer today (http://gco.iarc.fr/today/fact-sheets-cancers).

Zhou, Y., He, P., Xie, X., and Sun, C. 2019. Knockdown of SUMO1P3 represses tumor growth and invasion and enhances radio sensitivity in hepatocellular carcinoma. MOLECULAR AND

499 CELLULAR BIOCHEMISTRY 450:125-134. 10.1007/s11010-018-3379-8 


\section{Table 1 (on next page)}

Clinical parameters of patients in the training set and testing set

BCLC stage: Barcelona Clinic Liver Cancer stage; Multinodular: Whether the tumor has multiple nodules. 
1 Table 1. Clinical parameters of patients in the training set and testing set

\begin{tabular}{|c|c|c|c|}
\hline Variables & Training set(n=103) & Testing set(n=100) & $P$ value \\
\hline \multicolumn{4}{|l|}{ Gender } \\
\hline Female & 13 & 13 & \\
\hline Male & 90 & 87 & 1 \\
\hline \multicolumn{4}{|l|}{ Age } \\
\hline$<60$ & 80 & 81 & \\
\hline$\geq 60$ & 23 & 19 & 0.68 \\
\hline \multicolumn{4}{|l|}{ ALT } \\
\hline$<=50 \mathrm{U} / \mathrm{L}$ & 61 & 54 & \\
\hline$>50 \mathrm{U} / \mathrm{L}$ & 42 & 46 & 0.542 \\
\hline \multicolumn{4}{|l|}{ Main Tumor Size } \\
\hline$<=5 \mathrm{~cm}$ & 68 & 58 & \\
\hline$>5 \mathrm{~cm}$ & 34 & 42 & \\
\hline NA & 1 & 0 & 0.26 \\
\hline \multicolumn{4}{|l|}{ Multinodular } \\
\hline $\mathrm{NO}$ & 86 & 74 & \\
\hline YES & 17 & 26 & 0.138 \\
\hline \multicolumn{4}{|l|}{ TNM stage } \\
\hline $\mathrm{I}+\mathrm{II}$ & 82 & 71 & \\
\hline III & 21 & 27 & 0.305 \\
\hline NA & 0 & 2 & \\
\hline \multicolumn{4}{|l|}{ BCLC stage } \\
\hline $0+\mathrm{A}$ & 83 & 69 & \\
\hline $\mathrm{B}+\mathrm{C}$ & 20 & 29 & 0.13 \\
\hline NA & 0 & 2 & \\
\hline \multicolumn{4}{|l|}{ AFP } \\
\hline$<=300 \mathrm{ng} / \mathrm{ml}$ & 54 & 52 & \\
\hline$>300 \mathrm{ng} / \mathrm{ml}$ & 46 & 48 & 0.887 \\
\hline NA & 3 & 0 & \\
\hline
\end{tabular}

2 BCLC stage: Barcelona Clinic Liver Cancer stage; Multinodular: Whether the

3 tumor has multiple nodules 


\section{Table 2 (on next page)}

Five candidate IncRNAs screened by LASSO regression were inserted in multivariate COX regression. Four IncRNAs with independent prognostic significance for overall survival were screened out. Details of the four IncRNAs are as follows.

Correlation coefficient and Hazard ratio were the results of multivariate COX regression. 
1 Table 2. Five candidate IncRNAs screened by LASSO regression were inserted

2 in multivariate COX regression. Four IncRNAs with independent prognostic

3 significance for overall survival were screened out.

\begin{tabular}{llllll}
\hline Gene name & $\begin{array}{l}\text { Ensemble } \\
\text { ID }\end{array}$ & coefficient & $\begin{array}{l}\text { Hazard } \\
\text { ratio }\end{array}$ & P value & Chromosome \\
\hline AC093797. & ENSG0000 & -0.4818 & 0.6177 & 0.0003 & Chr4:185587909- \\
& 0233110 & & & & $185594004(+)$ \\
\hline AL121748.1 & ENSG0000 & 0.4404 & 1.5534 & 0.038 & Chr10:33211277- \\
& 0238258 & & & & $33213805(+)$ \\
\hline AL162231.4 & ENSG0000 & 1.2845 & 3.6129 & 0.0221 & Chr9:34661903- \\
& 0261215 & & & & $34666029(-)$ \\
\hline POLR2J4 & ENSG0000 & -1.517 & 0.2194 & 0.0081 & Chr7:43940895- \\
& 0214783 & & & & $44019175(-)$
\end{tabular}

5 Correlation coefficient and Hazard ratio were the results of multivariate COX

6 regression. 


\section{Table 3 (on next page)}

Stratified analysis of the overall survival of four-IncRNA signature in the training set and testing set.

*Statistically significant. Abbreviations: BCLC stage: Barcelona Clinic Liver Cancer stage; Multinodular: Whether the tumor has multiple nodules. $\mathrm{HR}$, Hazard ratio; $95 \% \mathrm{Cl}, 95 \%$ confidence interval. 
1 Table3.Stratified analysis of the overall survival of four-IncRNA signature in

2 the training set and testing set.

\begin{tabular}{lllllll}
\hline Variable & \multicolumn{2}{l}{ Training set } & \multicolumn{3}{c}{ Testing set } \\
\cline { 2 - 6 } & Number & HR & $P$ & Number & HR & $P$ \\
$($ High/Lo & $(95 \%$ CI $)$ & value & $($ High/Lo & $(95 \% C I)$ & value \\
w) & & & w) & \\
\hline
\end{tabular}

\section{Gender}

Female $\quad 6 / 7$

$3.939(0.40 \quad 0.2 \quad 6 / 7$

$4.037(0.36 \quad 0.22$

9-37.96)

$5-44.68)$

Male

$45 / 45$

$3.603(1.66 \quad 0.0005 \quad 38 / 49$

$2.405(1.31 \quad 0.0034$

8-7.781) *

2-4.408) *

Age

$<60$

$45 / 35$

$3.536(1.51$

$0.0019 \quad 34 / 47$

$2.412(1.26$

0.0056

2-8.27)

*

9-4.584) *

$\geq 60$

$6 / 17$

4.253(0.94

$0.059 \quad 10 / 9$

$7.153(0.85 \quad 0.069$

6-19.11)

8-59.62)
ALT
$<=50 \mathrm{U} / \mathrm{L} \quad 27 / 34$
$2.985(1.32$
$0.0056 \quad 22 / 32$
$2.479(1.04 \quad 0.033 *$
5-6.726) *
$7-5.868)$
$>50 \mathrm{U} / \mathrm{L} \quad 24 / 18$
$10.87(1.38 \quad 0.0045 \quad 22 / 24$
$2.358(1.05 \quad 0.032 *$ 


$6-85.23) \quad * \quad 6-5.269)$

Main Tumor Size

$\begin{array}{lllllll}<=5 \mathrm{~cm} & 32 / 36 & 5.949(1.96 & 0.0003 & 21 / 37 & 2.145(0.99 & 0.0523\end{array}$

$\left.3-18.02) \quad 6^{*} \quad 3-4.637\right)$

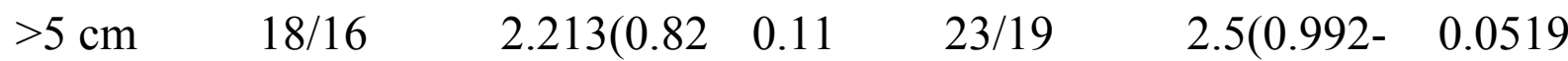

$5-5.94) \quad 6.298)$

Multinodular

\begin{tabular}{|c|c|c|c|c|c|c|}
\hline \multirow[t]{2}{*}{ NO } & $39 / 47$ & $4.157(1.83$ & 0.0002 & $30 / 44$ & $3.017(1.47$ & 0.0015 \\
\hline & & $4-9.419)$ & $1^{*}$ & & $2-6.183)$ & $*$ \\
\hline \multirow[t]{2}{*}{ YES } & $12 / 5$ & $1.575(0.31$ & 0.58 & $14 / 12$ & $0.641(0.23$ & 0.39 \\
\hline & & $6-7.857)$ & & & $1-1.777)$ & \\
\hline
\end{tabular}

TNM stage

$\begin{array}{lllllll}\text { I+II } & 37 / 45 & 4.186(1.63 & 0.0012 & 24 / 47 & 2.779(1.33 & 0.0043 \\ & & 3-10.73) & * & & 7-5.778) & * \\ \text { III } & 14 / 7 & 1.778(0.55 & 0.33 & 19 / 8 & 1.454(0.50 & 0.49 \\ & & & & & 3-4.206) & \end{array}$

BCLC stage

$\begin{array}{lllllll}0+\mathrm{A} & 36 / 47 & 4.728(1.85 & 0.0003 & 26 / 43 & 3.238(1.48 & 0.0019 \\ & & 8-12.03) & 3 * & & 1-7.08) & * \\ \mathrm{~B}+\mathrm{C} & 15 / 5 & 0.879(0.27 & 0.83 & 17 / 12 & 0.630(0.25 & 0.32\end{array}$




$$
5-2.816)
$$
AFP
$<=300$ ng/ $21 / 33$
$3.944(1.44$
0.0039
$17 / 35$
$4.625(1.84 \quad 0.0003$
$\mathrm{ml}$
6-10.76) *
2-11.61) $\quad 7^{*}$
$>300 \mathrm{ng} / \mathrm{m} \quad 29 / 17$
$3.012(1.00$
$0.039 * 27 / 21$
$1.438(0.65 \quad 0.36$
1
4-9.033)
9-3.136)

*Statistically significant. Abbreviations: BCLC stage: Barcelona Clinic Liver

Cancer stage; Multinodular: Whether the tumor has multiple nodules. HR, 5 Hazard ratio; $\mathbf{9 5 \%}$ CI, $\mathbf{9 5} \%$ confidence interval. 


\section{Table 4 (on next page)}

Stratified analysis of the recurrence-free survival of four-IncRNA signature in the training set and testing set.

*Statistically significant. Abbreviations: BCLC stage: Barcelona Clinic Liver Cancer stage; Multinodular: Whether the tumor has multiple nodules. HR, Hazard ratio; $95 \% \mathrm{Cl}, 95 \%$ confidence interval. 
1 Table4.Stratified analysis of the recurrence-free survival of four-IncRNA

2 signature in the training set and testing set.

\begin{tabular}{lllllll}
\hline Variable & \multicolumn{2}{l}{ Training set } & \multicolumn{4}{c}{ Testing set } \\
\cline { 2 - 6 } & Number & HR & $P$ & Number & HR & $P$ \\
$($ High/Lo & $(95 \%$ CI $)$ & value & $($ High/Lo & $(95 \%$ CI $)$ & value \\
w) & & & w) & & \\
\hline
\end{tabular}

\section{Gender}

Female $\quad 6 / 7$

$5.765(0.64 \quad 0.078 \quad 6 / 7$

$7.811(0.80 \quad 0.077$

$-51.95)$

$1-76.15)$

Male

$45 / 45$

$2.166(1.21$

$0.0071 \quad 38 / 49$

$2.072(1.22$

$0.0056^{*}$

7-3.854) *

Age

$<60$

$45 / 35$

$2.34(1.214$

$0.0089 \quad 34 / 47$

1.954(1.10

$0.02 *$

$-4.51)$

2-3.464)

$\geq 60$

$6 / 17$

$3.758(1.14$

$0.019 * 9 / 9$

$4.428(1.19$

$0.015^{*}$

9-12.29)

$-16.47)$
ALT
$<=50 \mathrm{U} / \mathrm{L} \quad 27 / 34$
$2.164\left(1.08 \quad 0.026^{*} \quad 22 / 32\right.$
$1.974(0.94 \quad 0.064$
$-4.333)$
7-4.115)
$>50 \mathrm{U} / \mathrm{L} \quad 24 / 18$
$2.852(1.10 \quad 0.024 * 22 / 24$
2.394(1.17
$0.013^{*}$ 
2-7.379)

$9-4.86)$

Main Tumor Size

\begin{tabular}{|c|c|c|c|c|c|c|}
\hline \multirow[t]{2}{*}{$<=5 \mathrm{~cm}$} & $32 / 36$ & $3.655(1.72$ & 0.0003 & $21 / 37$ & $1.95(1.018$ & $0.04^{*}$ \\
\hline & & $5-7.745)$ & $*$ & & $-3.735)$ & \\
\hline \multirow[t]{2}{*}{$>5 \mathrm{~cm}$} & $18 / 16$ & $1.324(0.57$ & 0.51 & $23 / 19$ & $2.586(1.07$ & $0.028 *$ \\
\hline & & $1-3.07)$ & & & $9-6.199)$ & \\
\hline
\end{tabular}

Multinodular

$\begin{array}{lllllll}\text { NO } & 39 / 47 & 2.514(1.38 & 0.0018 & 30 / 44 & 2.516(1.37 & 0.0019 * \\ & & 2-4.573) & * & & 9-4.592) & \\ \text { YES } & 12 / 5 & 1.596(0.32 & 0.56 & 14 / 12 & 1.658(0.63 & 0.3 \\ & & 9-7.749) & & & 5-4.331) & \end{array}$

TNM stage

$\begin{array}{lllllll}\text { I+II } & 37 / 45 & 2.608(1.35 & 0.0028 & 24 / 47 & 2.837(1.52 & 0.00061 \\ & & 7-5.01) & * & & 4-5.282) & * \\ \text { III } & 14 / 7 & 1.214(0.42 & 0.72 & 19 / 8 & 1.167(0.42 & 0.77 \\ & & & & & 8-3.184) & \end{array}$

BCLC stage

\begin{tabular}{lllllll}
$0+\mathrm{A}$ & $36 / 47$ & $2.658(1.38$ & 0.0023 & $26 / 43$ & $2.671(1.41$ & $0.0018^{*}$ \\
& & $5-5.104)$ & $*$ & & $-5.058)$ & \\
$\mathrm{B}+\mathrm{C}$ & $15 / 5$ & $0.564(0.18$ & 0.3 & $17 / 12$ & $1.608(0.66$ & 0.29 \\
\hline
\end{tabular}




$$
\text { 7-1.7) }
$$

$\begin{array}{lllllll}\text { AFP } & & & & & \\ <=300 \mathrm{ng} / & 21 / 33 & 2.228(1.07 & 0.027 * & 17 / 35 & 3.615(1.69 & 0.00042 \\ \mathrm{ml} & & 3-4.625) & & & 2-7.724) & * \\ >300 \mathrm{ng} / \mathrm{m} & 29 / 17 & 2.489(0.99 & 0.0524 & 27 / 21 & 1.426(0.70 & 0.32 \\ 1 & & 1-6.255) & & & 4-2.89) & \end{array}$

*Statistically significant. Abbreviations: BCLC stage: Barcelona Clinic Liver

Cancer stage; Multinodular: Whether the tumor has multiple nodules. HR, Hazard ratio; 95\%CI, 95\% confidence interval. 
Figure 1

Analysis of flowchart

The flowchart indicates the exploration process and potential mechanism of cirrhotic HCC prognostic IncRNAs. 


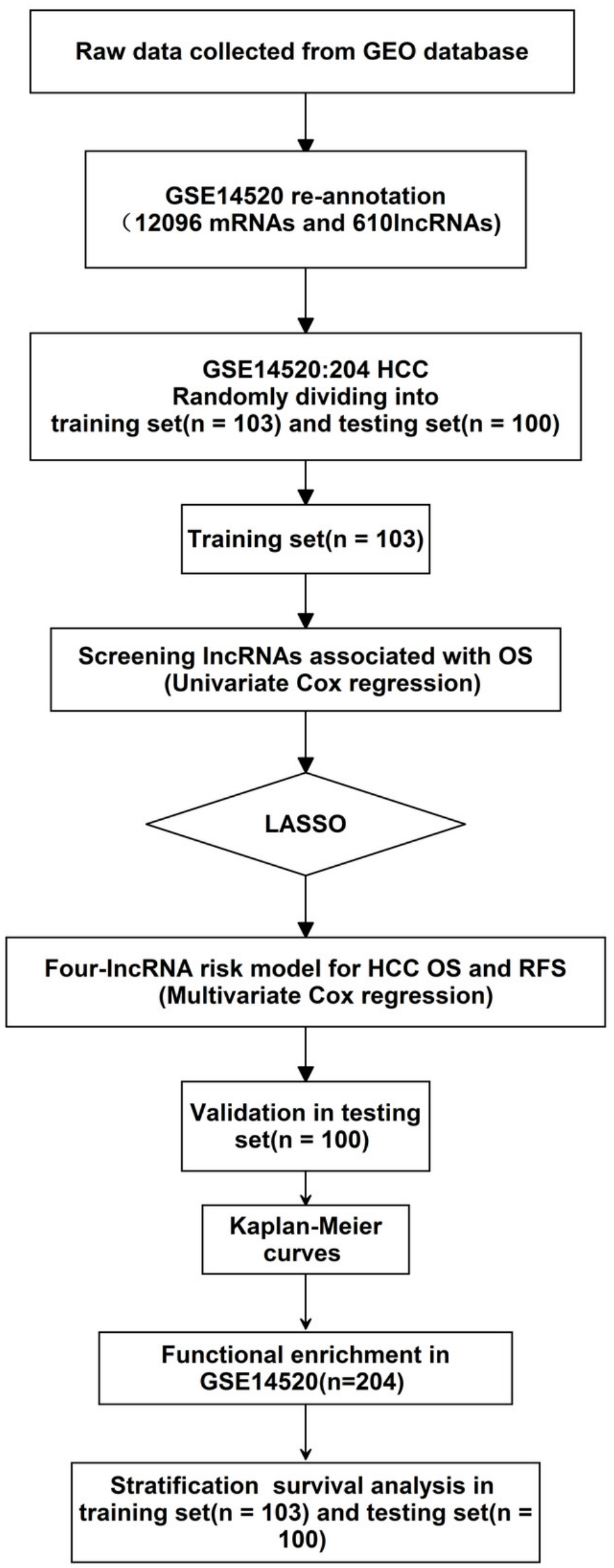


Figure 2

The remaining InCRNAs were further selected using LASSO regression analysis, and cross-validation was used to select the penalty parameters.

(A) LASSO coefficient profiles of the 35 candidate IncRNAs. Ten-time cross-validation for penalty parameters selection in the LASSO model. (B) A vertical line is drawn at the value chosen by 10 -fold cross-validation.
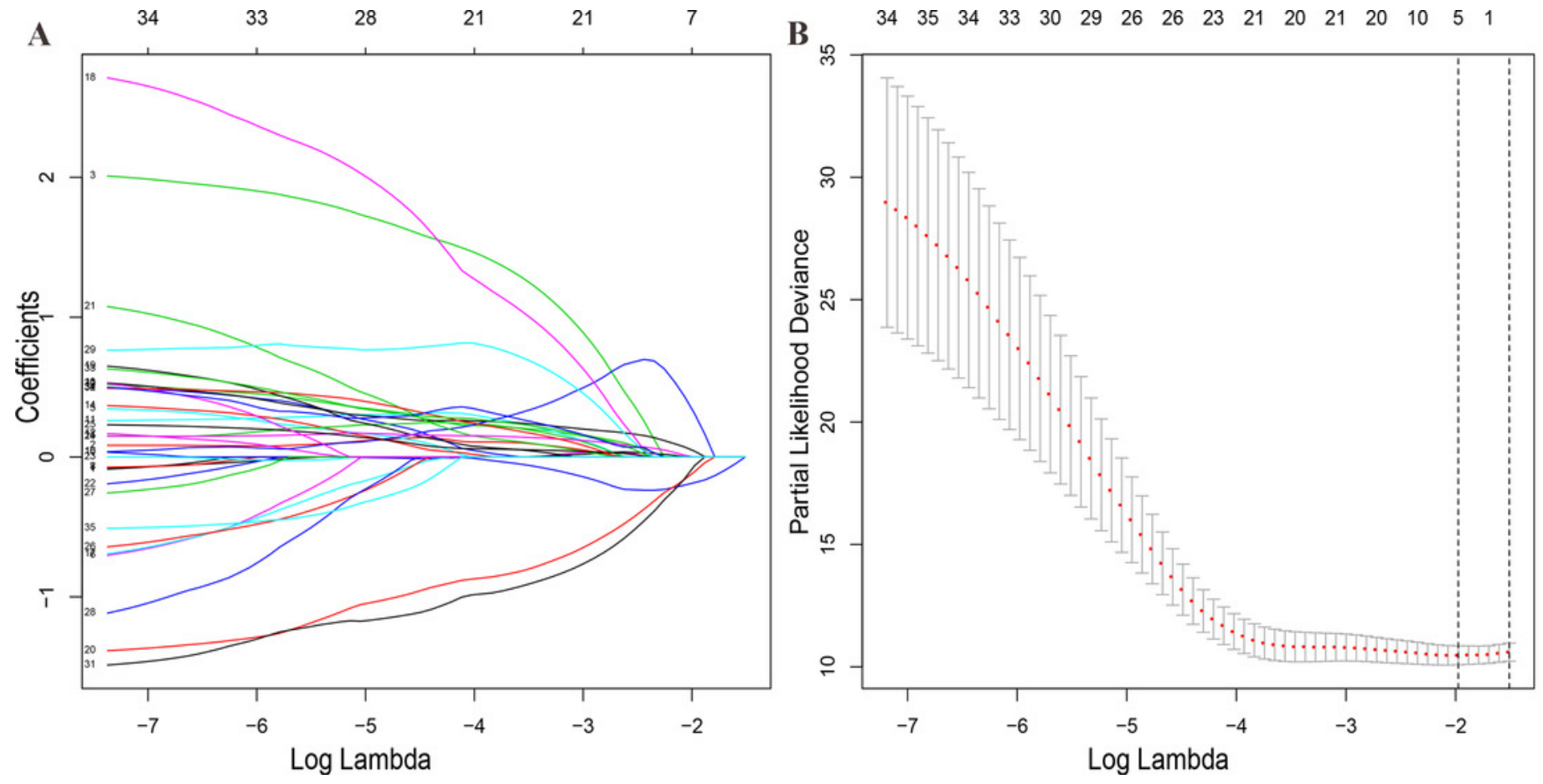
Figure 3

Prognostic efficiency of the prognostic risk scoring system for overall survival and recurrence-free survival.

(A) The four-IncRNA risk score distribution. (B) The heatmap of four IncRNAs gene expression in the high-risk and low-risk groups for the training set. (C) Overall survival status of patients in training set. (D) Recurrence status of patients in training set. 
$\mathbf{A}$

Training $\operatorname{set}(n=103)$

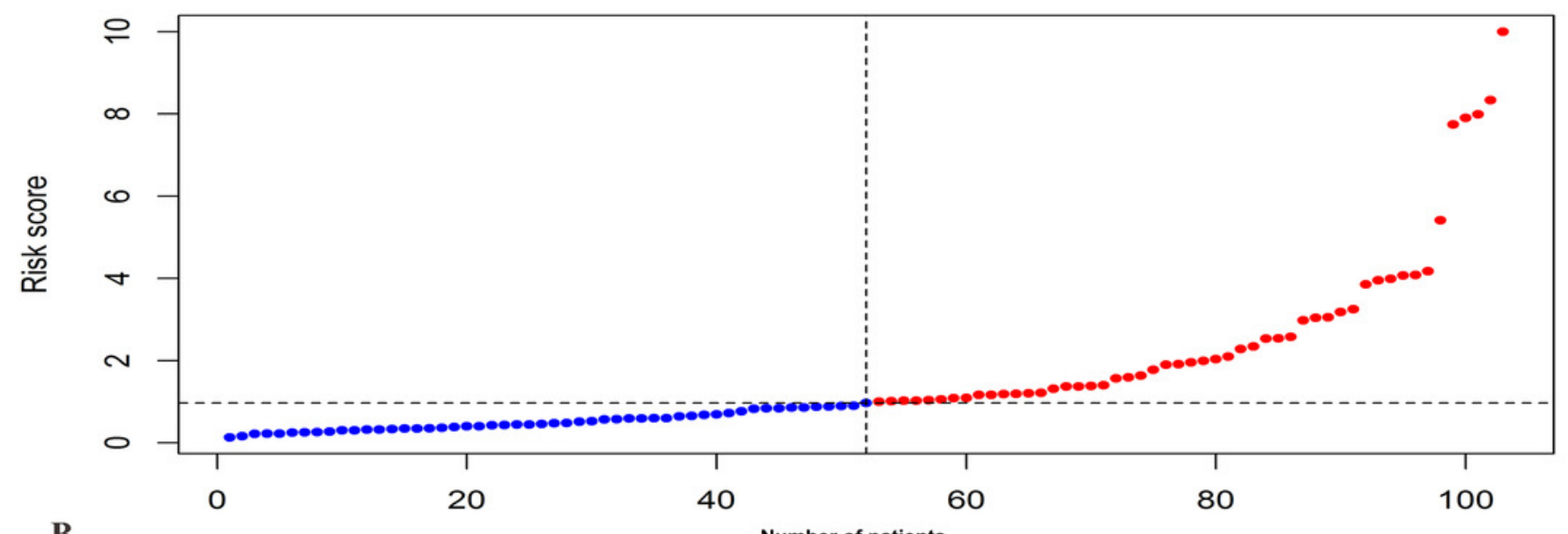

B

Number of patients
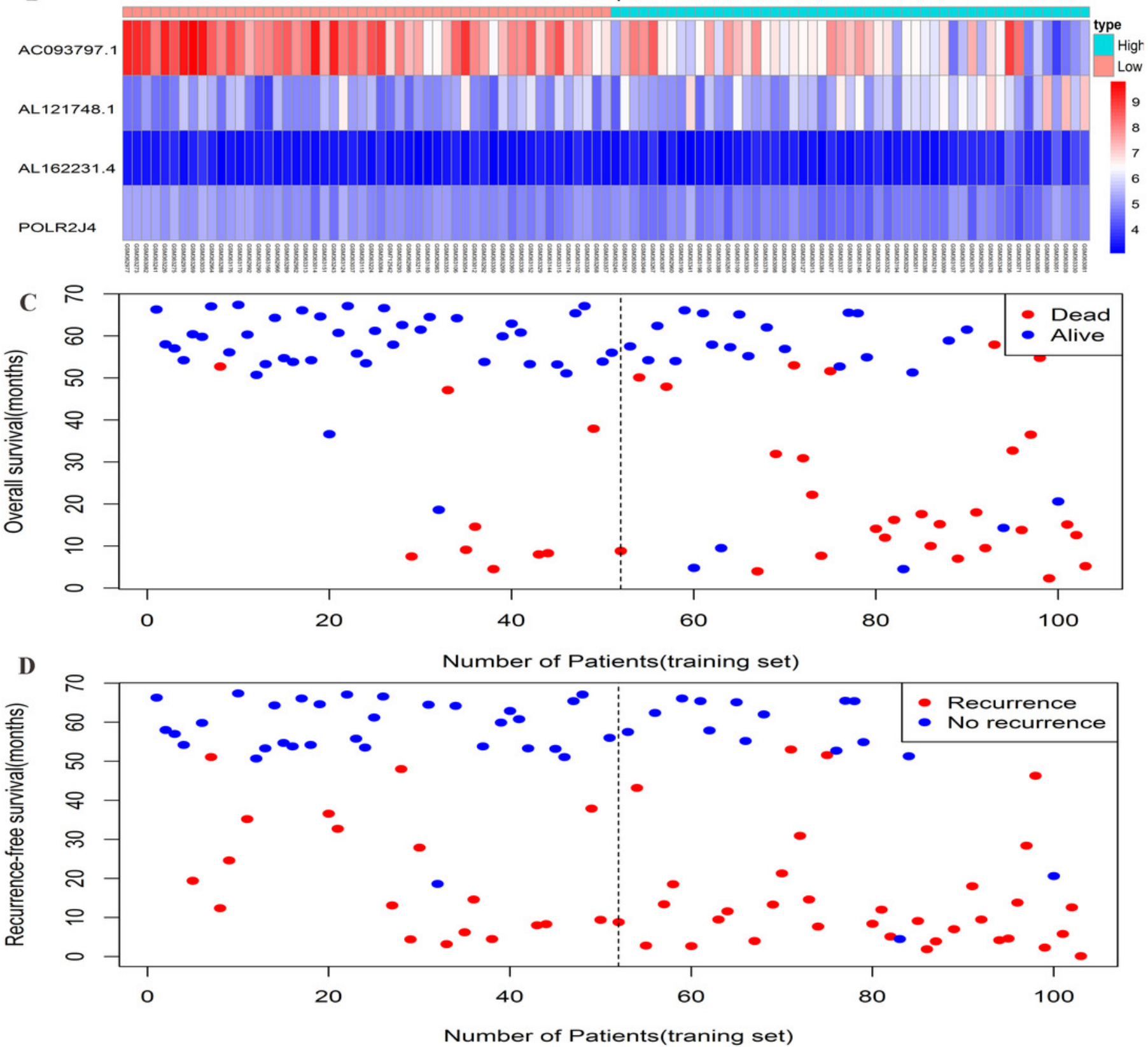

PeerJ reviewing PDF | (2019:04:36386:1:1:REVIEW 21 Jun 2019) 
Figure 4

Prognostic significance evaluation of four-IncRNA signature for overall survival (OS) and recurrence-free survival (RFS) in the training set.

Kaplan-Meier analysis for high-risk and low-risk groups was used to analyze OS and RFS. (A) The survival curve of OS. (B) The survival curve of RFS. (C)ROC curve for prognosis prediction of the risk score model at 5 years of OS. (D) ROC curve at 5 years of RFS. 

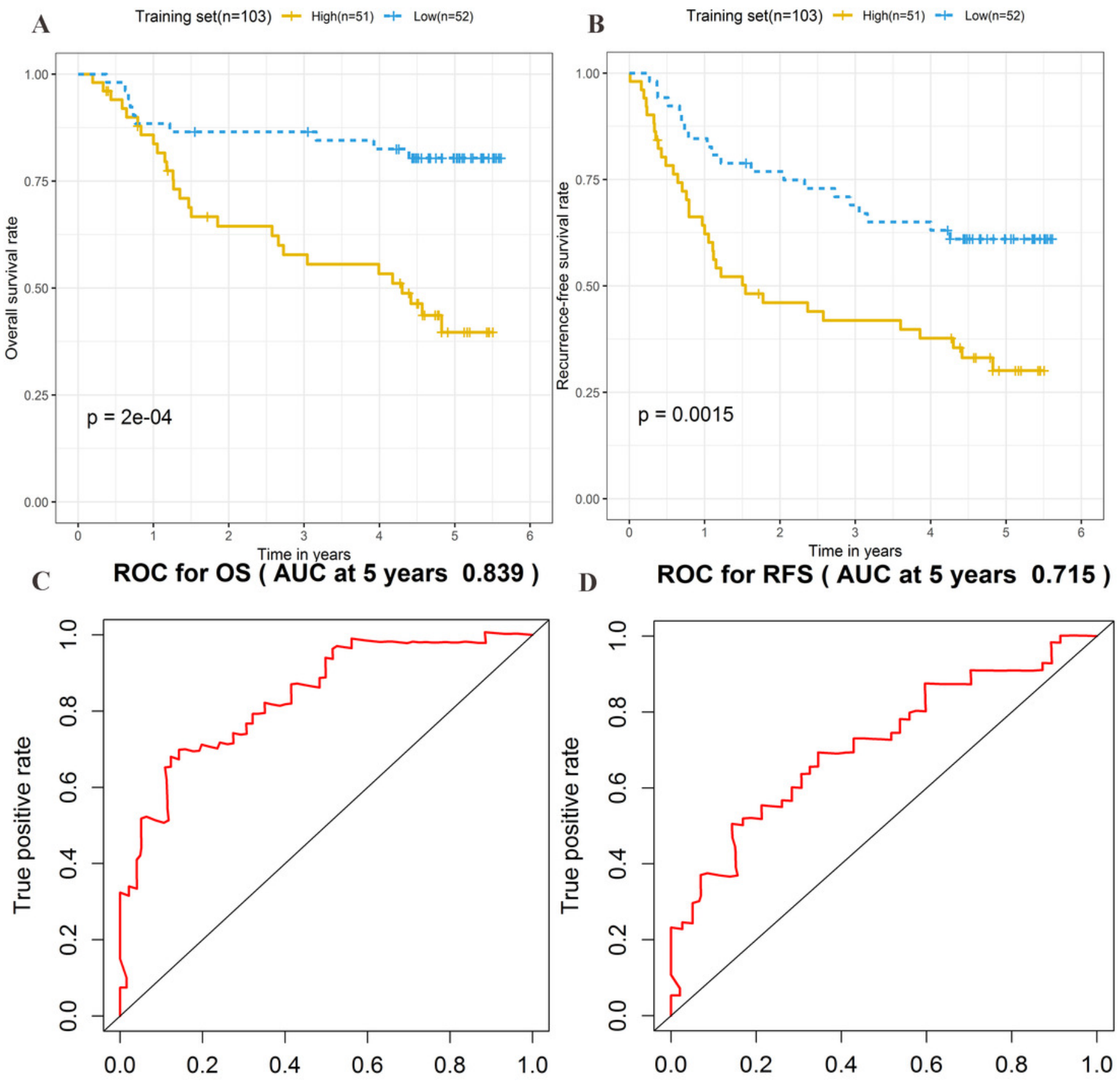

False positive rate

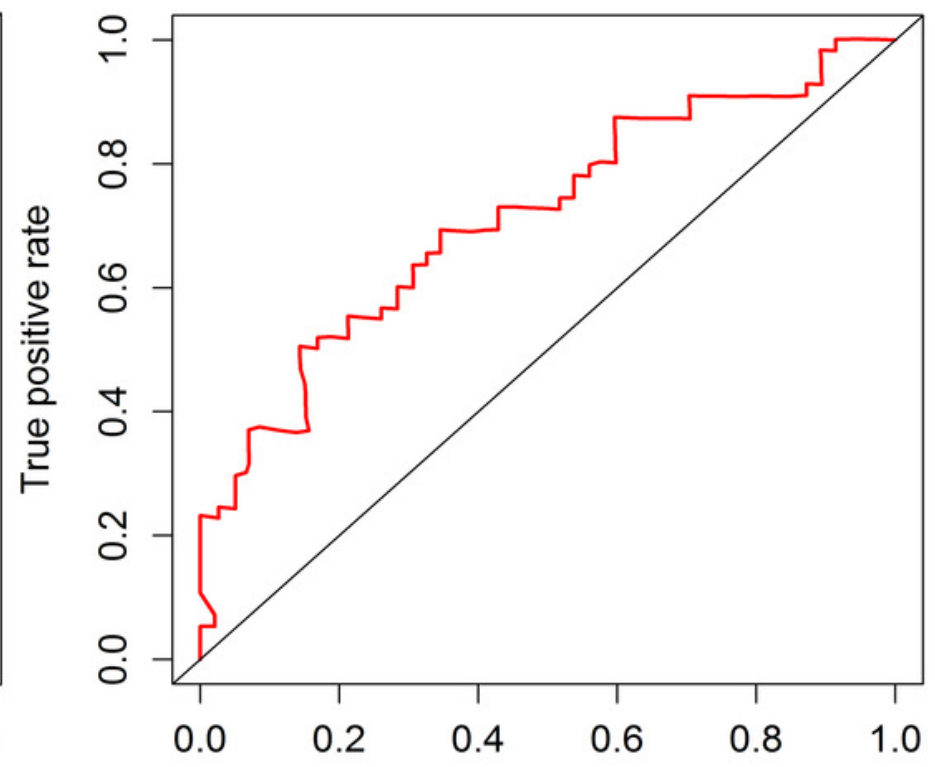

False positive rate 


\section{Figure 5}

Prognostic performance of four-IncRNA signature on overall survival(OS) and recurrence-free survival(RFS) in the testing set.

Kaplan-Meier analysis for high-risk and low-risk groups was used to analyze overall survival and recurrence-free survival. (A) The survival curve of OS. (B) The survival curve of RFS. (C)ROC curve for prognosis prediction of the risk score model at 5 years of OS. (D) ROC curve at 5 years of RFS.(E) Overall survival status of patients in testing set. (F) Recurrence status of patients in testing set. 

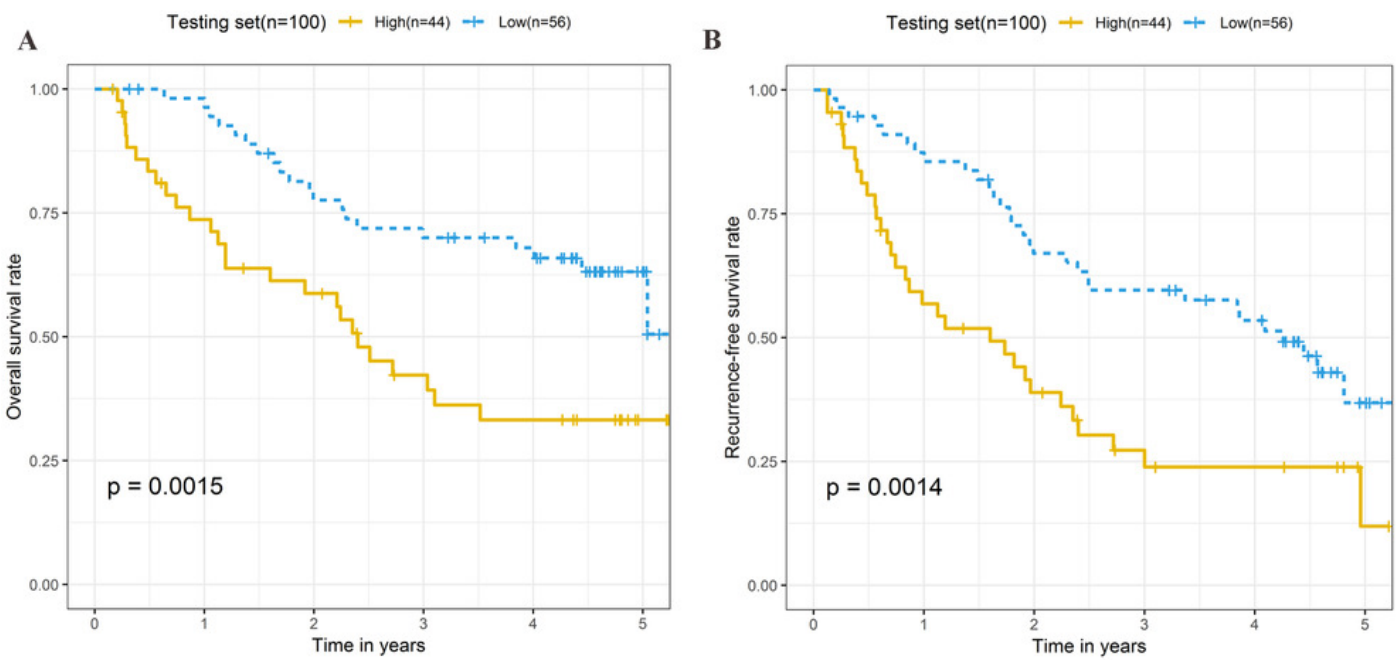

C ROC for OS ( AUC at 5 years 0.757 )
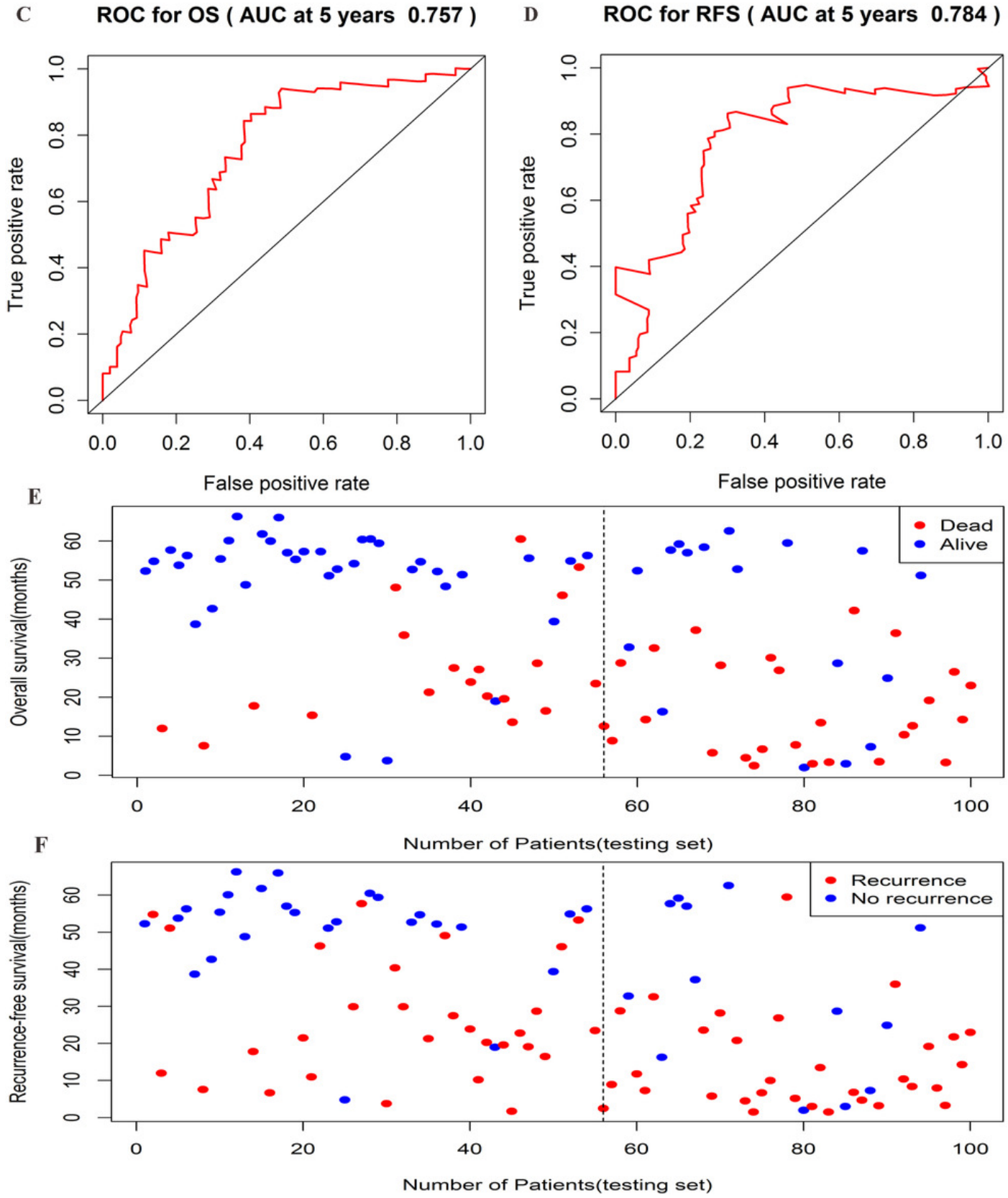

PeerJ reviewing PDF | (2019:04:36386:1:1:REVIEW 21 Jun 2019) 


\section{Figure 6}

Kaplan-Meier curves of the overall survival for high-risk and low-risk patients with different clinical characteristics in training set and testing set.

Following clinical characteristics :(A) solitary tumor in training set.(B) solitary tumor in testing set. (C) TNM stage I-II in training set. (D) TNM stage I-II in testing set. (E) BCLC stage 0-A in training set. (F) BCLC stage 0-A in testing set. 
$\mathbf{A}$

Training set:solidary tumor $(n=86)+\operatorname{High}(n=39)+\operatorname{Low}(n=47)$

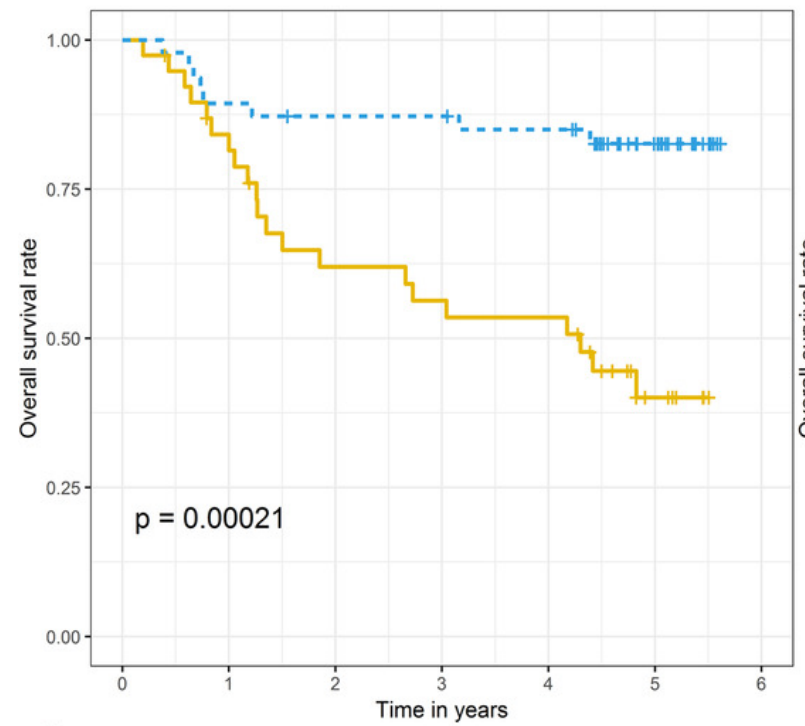

C

Training set:TNM stage $\mathrm{I}-\mathrm{II}(\mathrm{n}=82)+\operatorname{High}(\mathrm{n}=37)+\operatorname{Low}(\mathrm{n}=45)$

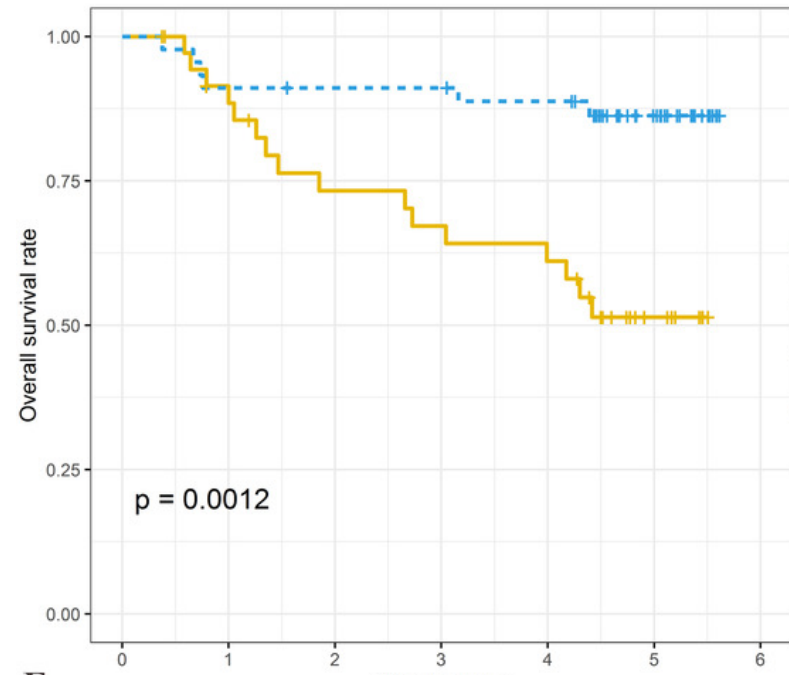

E

Training set:BCLC stage $0-A(n=83)+H i g h(n=36)+$ Low $(n=47)$

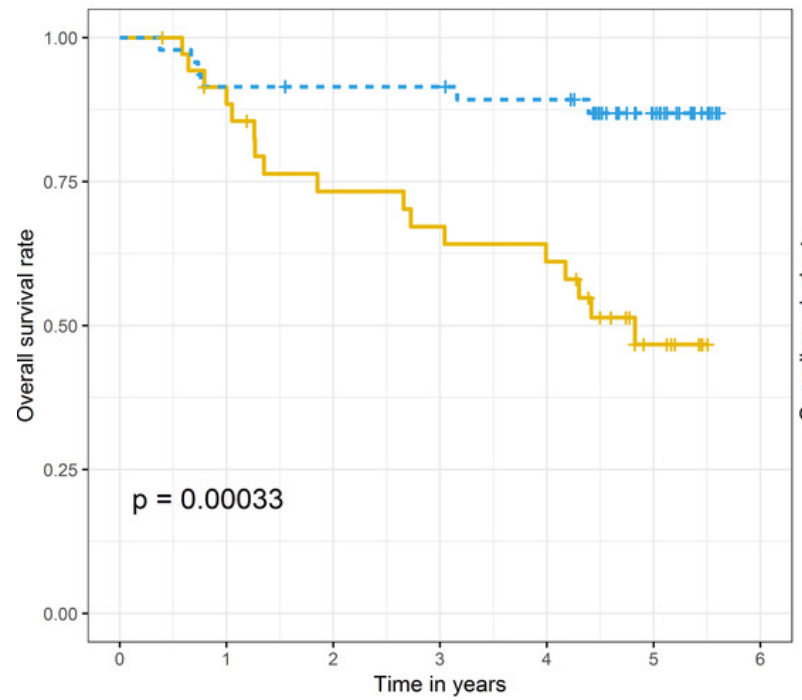

B

Testing set:solidary tumor $(n=74)+\operatorname{High}(n=30)+\operatorname{Low}(n=44)$

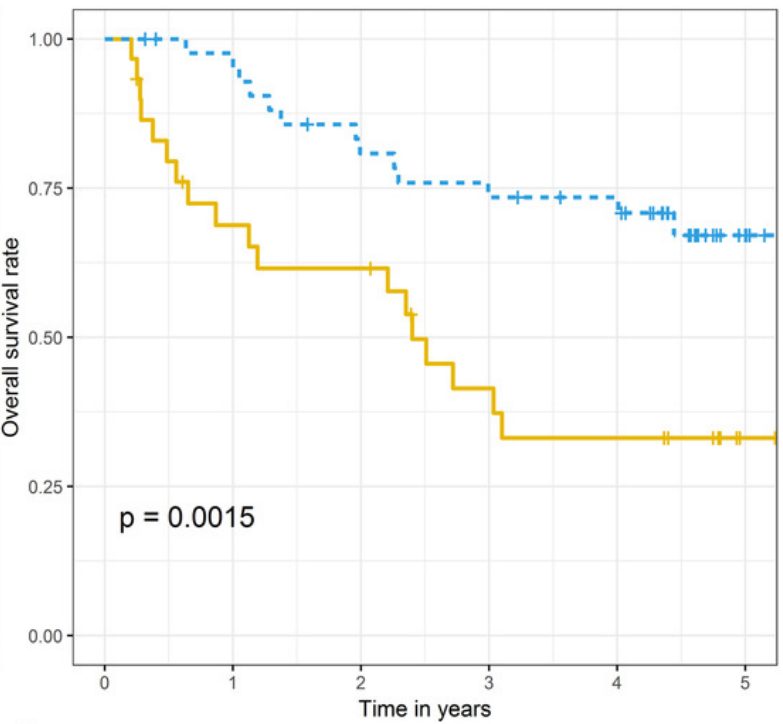

D

Testing set:TNM stage I-II(n=71) $+\operatorname{High}(n=24)+\operatorname{Low}(n=47)$

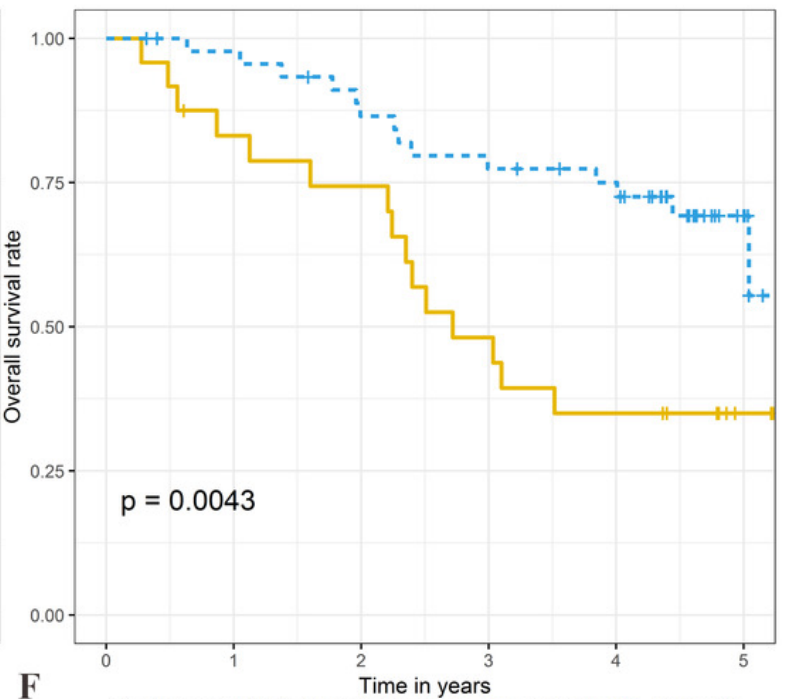

Testing set:BCLC stage $0-A(n=69)+\operatorname{High}(n=26)+-\operatorname{Low}(n=43)$

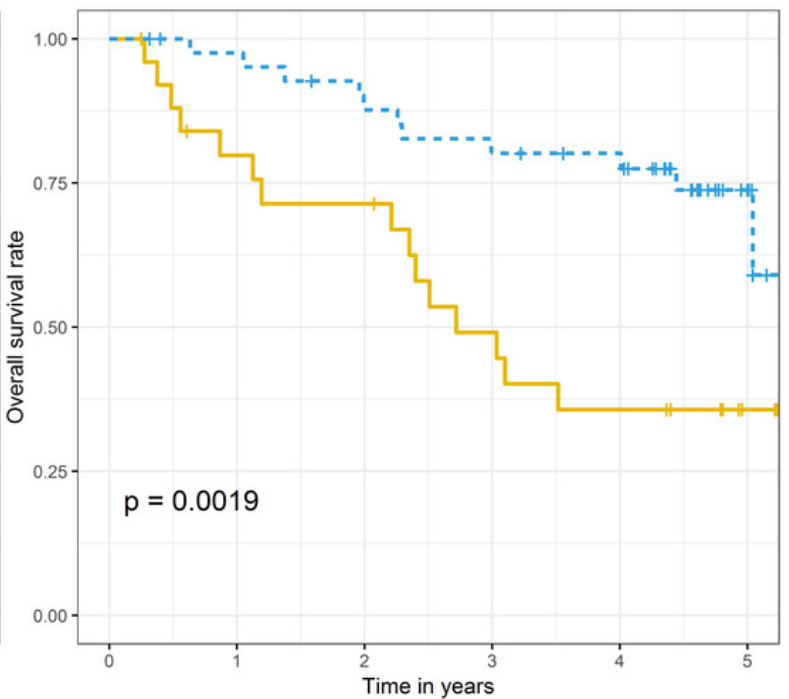




\section{Figure 7}

Kaplan-Meier curves of the recurrence-free survival for high-risk and low-risk patients with different clinical characteristics in training set and testing set.

Following clinical characteristics :(A) solitary tumor in training set.(B) solitary tumor in testing set. (C) TNM stage I-II in training set. (D) TNM stage I-II in testing set. (E) BCLC stage 0-A in training set. (F) BCLC stage 0-A in testing set. 
A Training set:solidary tumor $(n=86)+\operatorname{High}(n=39)+\operatorname{Low}(n=47)$

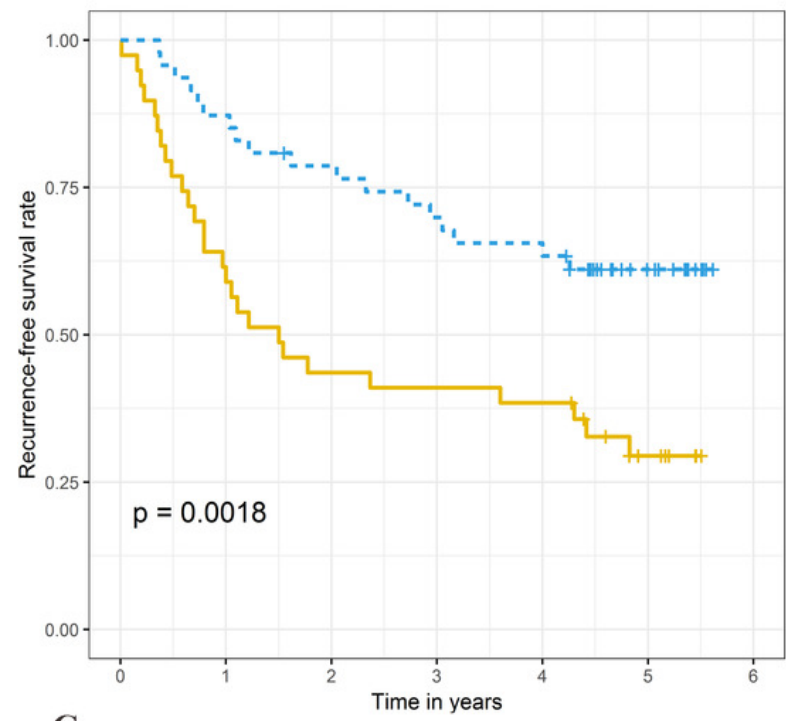

C

Training set:TNM stage $I-1 \mid(n=82)+H i g h(n=37)+\quad$ Low $(n=45)$

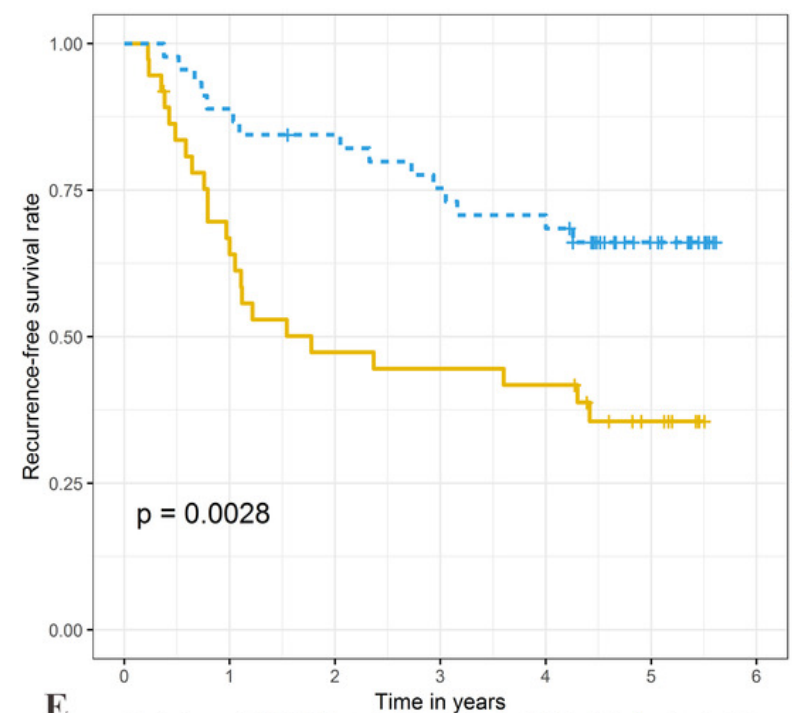

$\mathbf{E}$

Training set:BCLC stage $0-A(n=83)+\operatorname{High}(n=36)+\operatorname{Low}(n=47)$

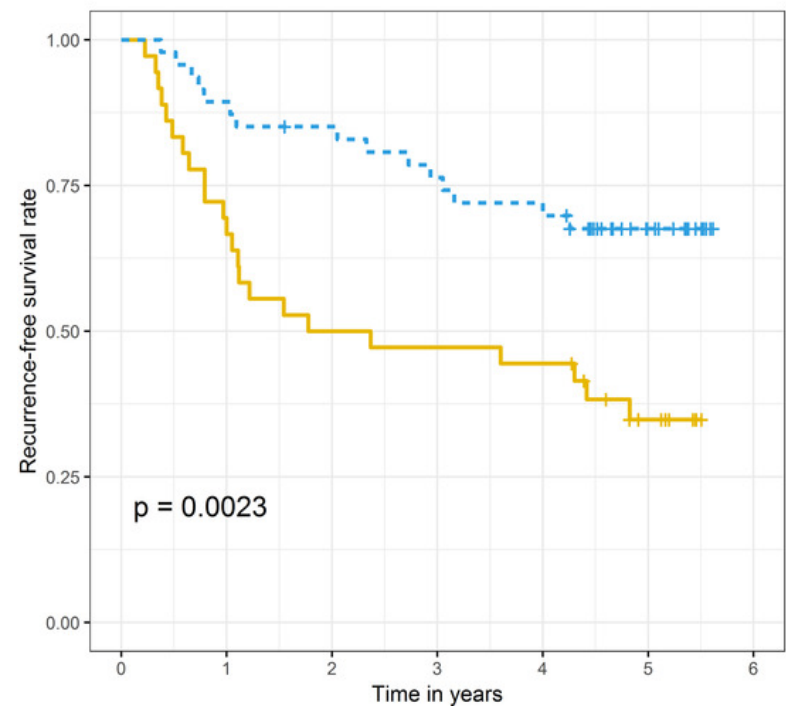

B Testing set:solitary tumor $(n=74)+\operatorname{High}(n=30)+\operatorname{Low}(n=44)$

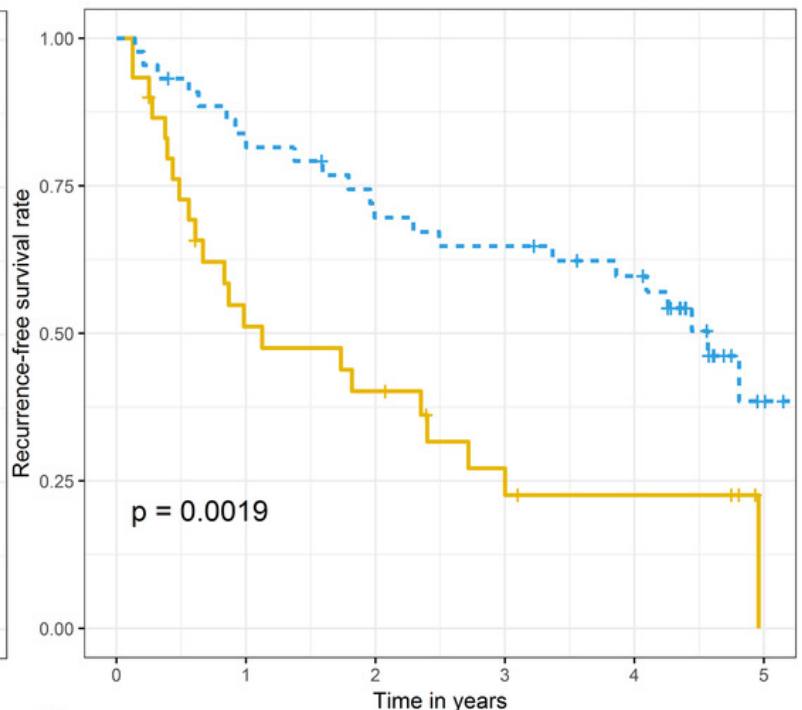

D

Testing set:TNM stage I-II(n=71) $+\operatorname{High}(n=24)+\operatorname{Low}(n=47)$

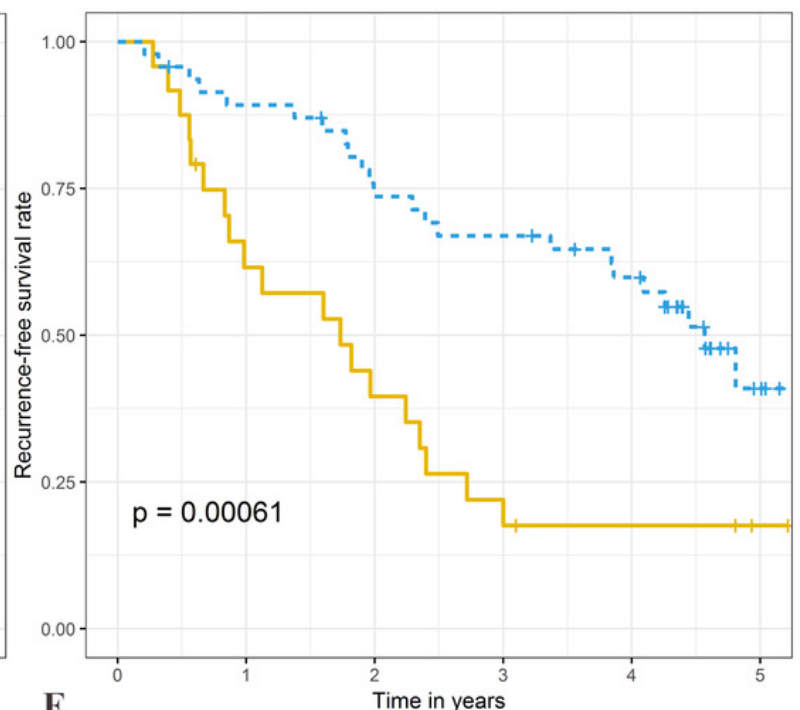

F Testing set:BCLC stage $0-A(n=69)+H i g h(n=26)+\operatorname{Low}(n=43)$

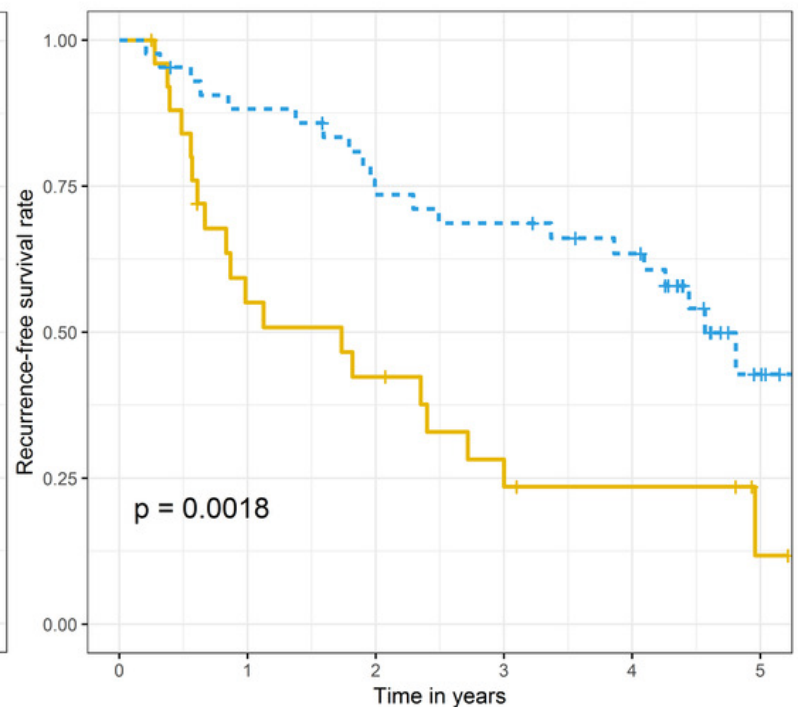


Figure 8

Functional enrichment analysis of four IncRNAs-associated protein-coding genes.

(A) Significantly enriched top $25 \mathrm{GO}$ (gene ontology) terms. (B) Top $25 \mathrm{KEGG(Kyoto}$ Encyclopedia of Genes and Genomes) pathways. 
A

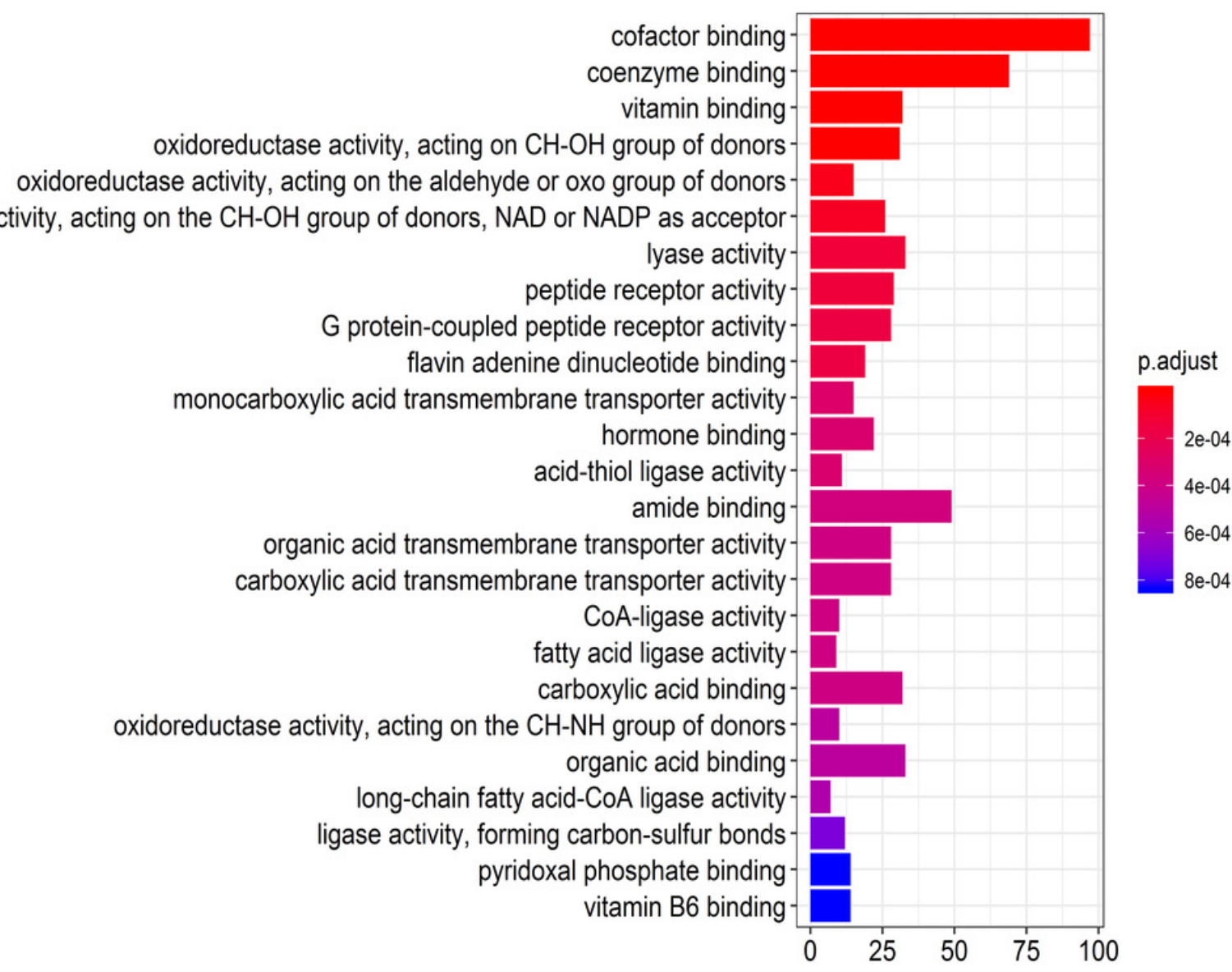

B

Neuroactive ligand-receptor interaction Carbon metabolism Fatty acid degradation Peroxisome Valine, leucine and isoleucine degradation PPAR signaling pathway Chemical carcinogenesis Lysine degradation Metabolism of xenobiotics by cytochrome P450 Glycolysis / Gluconeogenesis Drug metabolism - cytochrome P450 Glycine, serine and threonine metabolism

Tryptophan metabolism Bile secretion Adipocytokine signaling pathway beta-Alanine metabolism Fatty acid metabolism Tyrosine metabolism Arginine and proline metabolism Propanoate metabolism Pyruvate metabolism

Butanoate metabolism Carbohydrate digestion and absorption Glyoxylate and dicarboxylate metabolism Histidine metabolism

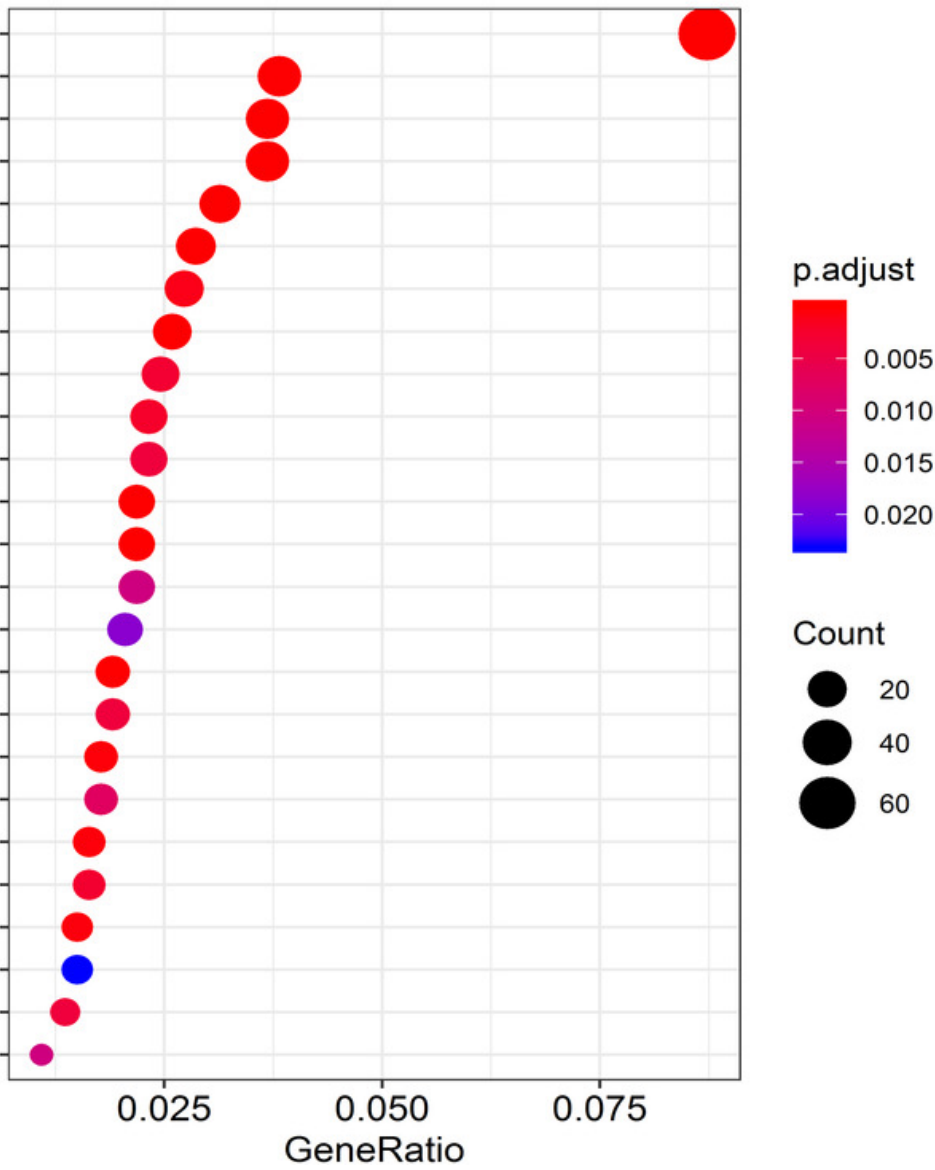

